Research Article

\title{
Existence and Stability for a Nonlinear Coupled $p$-Laplacian System of Fractional Differential Equations
}

\author{
Merfat Basha $\mathbb{D}^{1,2}$ Binxiang Dai $\mathbb{D D}^{1}$ and Wadhah Al-Sadi $\mathbb{D}^{3}$ \\ ${ }^{1}$ School of Mathematics and Statistics, Central South University, Changsha 410085, China \\ ${ }^{2}$ Department of Mathematics and Computer, College of Science, Ibb University, Ibb, Yemen \\ ${ }^{3}$ School of Mathematics and Physics, China University of Geosciences, Wuhan, China \\ Correspondence should be addressed to Merfat Basha; merfat2019@csu.edu.cn
}

Received 28 December 2020; Revised 4 February 2021; Accepted 11 February 2021; Published 26 February 2021

Academic Editor: Ahmet Ocak Akdemir

Copyright (@ 2021 Merfat Basha et al. This is an open access article distributed under the Creative Commons Attribution License, which permits unrestricted use, distribution, and reproduction in any medium, provided the original work is properly cited.

\begin{abstract}
In this paper, we study the nonlinear coupled system of equations with fractional integral boundary conditions involving the Caputo fractional derivative of orders $\theta_{1}$ and $\theta_{2}$ and Riemann-Liouville derivative of orders $\varrho_{1}$ and $\varrho_{2}$ with the $p$-Laplacian operator, where $n-1<\theta_{1}, \theta_{2}, \varrho_{1}, \varrho_{2} \leq n$, and $n \geq 3$. With the help of two Green's functions $\left(G^{\varrho_{1}}(w, \Im), G^{\mathrm{e}_{2}}(w\right.$, $\left.\mathfrak{\Im})\right)$, the considered coupled system is changed to an integral system. Since topological degree theory is more applicable in nonlinear dynamical problems, the existence and uniqueness of the suggested coupled system are treated using this technique, and we find appropriate conditions for positive solutions to the proposed problem. Moreover, necessary conditions are highlighted for the Hyer-Ulam stability of the solution for the specified fractional differential problems. To confirm the theoretical analysis, we provide an example at the end.
\end{abstract}

\section{Introduction}

The theoretical development of fractional calculus and its applications is more important to model nonlinear complex problems with the arbitrary fractional order. The subject of fractional differential equations (FDEs) has become an important area in real life because of their ability to model a lot of physical phenomena associated with rapid and concise changes with their significance in science and engineering through the past three decades, such as chemistry, physics, biology, engineering, visco-elasticity, electrotechnical, signal processing, electrochemistry, and controllability (see the details, [1-9], and the reference therein). In the near time, the nonlinear fractional partial differential equations are the most applied research area in which most authors and scientists are focused for their investigation. In this case, the Caputo derivative plays a great role to analyze the specific application of nonlinear PDEs. in [10], the authors have studied the cancer treatment model based on Caputo-Fabrizio fractional derivative. After integrating the model into the Caputo-Fabrizio fractional derivative, they have analyzed the existence of the solution as well. The Caputo-Fabrizio fractional derivative is implemented in [11] for the modeling and characterizing of the alcoholism. By applying the fixed-point theorem, they have studied the existence and uniqueness of the alcoholism model. The spread of the SIQR model is investigated by [12] using the Caputo derivative. They have justified the stability and uniqueness of the nonvirus equilibrium and virus equilibrium point.

For this problem, different authors proposed different numerical solution techniques. The analysis with the nonlinear time-fractional HIV/AIDS transmission model is considered in [13], in which the numerical solution is found using the fractional variational iteration method with convergence analysis. The nonlinear garden equation is studied in [14] based on the Atangana-Baleau Caputo derivative. He has highlighted the fixed-point theorem for proving the existence and uniqueness of the garden equation.

One of the main difficulties for the solution of the nonlinear fractional PDEs is to analyze the existence theory of solutions. Sufficient conditions for the existence and 
uniqueness of solutions (EUS) have been obtained by using different nonlinear analysis techniques and fixed-point theorems (for more details, read [15-18]). Also, the boundary value problems with various boundary conditions for many ordinary differential equations are studied [19-23]. However, the theory of boundary value problems for nonlinear FDEs is still not discussed more, and many problems of this theory require to be explored. On the contrary, the investigation of coupled systems of the differential equations is also significant because systems of this kind appear in various applied nature problems (refer [24-28]).

The topological degree theory is a useful tool in nonlinear analysis with numerous applications to operatorial equations, optimization theory, fractal theory, and other topics. We will see the following consideration of topological degree theory with boundary conditions based on the Caputo fractional derivative by different authors. Isaia [29] applied the topological degree theory to establish sufficient conditions for the existence of a solution for the following nonlinear integral equations:

$$
\pi(w)=\mathscr{Z}(w, \pi(w))+\int_{a}^{b} \mathscr{Q}(w, \mathfrak{I}, \pi(\mathfrak{I})) \mathrm{d} \mathfrak{I}, \quad w \in[a, b],
$$

where $\mathscr{Z}:[a, b] \times I R \longrightarrow I R$ and $Q$ : $[a, b] \times[a, b] \times$ $I R \longrightarrow I R$ are continuous functions. In their study [30],
Wang et al. used the topological degree method to obtain some existence conditions of the solution for the following nonlocal Cauchy problem:

$$
\left\{\begin{array}{l}
{ }^{c} D^{\varrho} \pi(w)=\mathscr{Z}(w, \pi(w)), \quad 0 \leq w \leq W, \\
\pi(0)+h(\pi)=\pi_{0},
\end{array}\right.
$$

where ${ }^{c} D^{\mathrm{e}}$ denotes the Caputo fractional derivative with order $\varrho \in(0,1)$ and $\mathscr{Z}: C([0, w], \mathbb{R}) \longrightarrow \mathbb{R}$ and $\pi_{0} \in \mathbb{R}$ are continuous. The nonlocal term $h: C([0, w], \mathbb{R}) \longrightarrow \mathbb{R}$ is a given function. Proceeding on the same fashion, Shah and Khan [31] proved the EUS for a coupled system under the fractional derivatives by using the technique of degree theory given as follows:

$$
\left\{\begin{array}{l}
{ }^{c} D^{\varrho_{1}} \pi_{1}(w)=\mathscr{Z}_{1}\left(w, \pi_{1}(w), \pi_{2}(w)\right), \quad w \in[0,1], \\
{ }^{c} D^{\varrho_{2}} \pi_{2}(w)=\mathscr{Z}_{2}\left(w, \pi_{1}(w), \pi_{2}(w)\right), \quad w \in[0,1], \\
\alpha_{1} \pi_{1}(0)-\beta_{1} \pi_{1}(\theta)-\delta_{1} \pi_{1}(1)=\phi_{1}\left(\pi_{1}\right), \\
\alpha_{2} \pi_{2}(0)-\beta_{2} \pi_{2}(\vartheta)-\delta_{2} \pi_{2}(1)=\phi_{2}\left(\pi_{2}\right),
\end{array}\right.
$$

where $\varrho_{1}, \varrho_{2}, \theta, \vartheta \in(0,1), \mathscr{Z}_{1}, \mathscr{Z}_{2} \in[0,1] \times R^{2} \longrightarrow R$, and $\phi_{1}, \phi_{2}: C([0,1], \mathbb{R}) \longrightarrow \mathbb{R}$ are continuous. Khan et al. [32] used the above-mentioned method to study the following coupled system in the sense of Caputo derivatives with p-Laplacian:

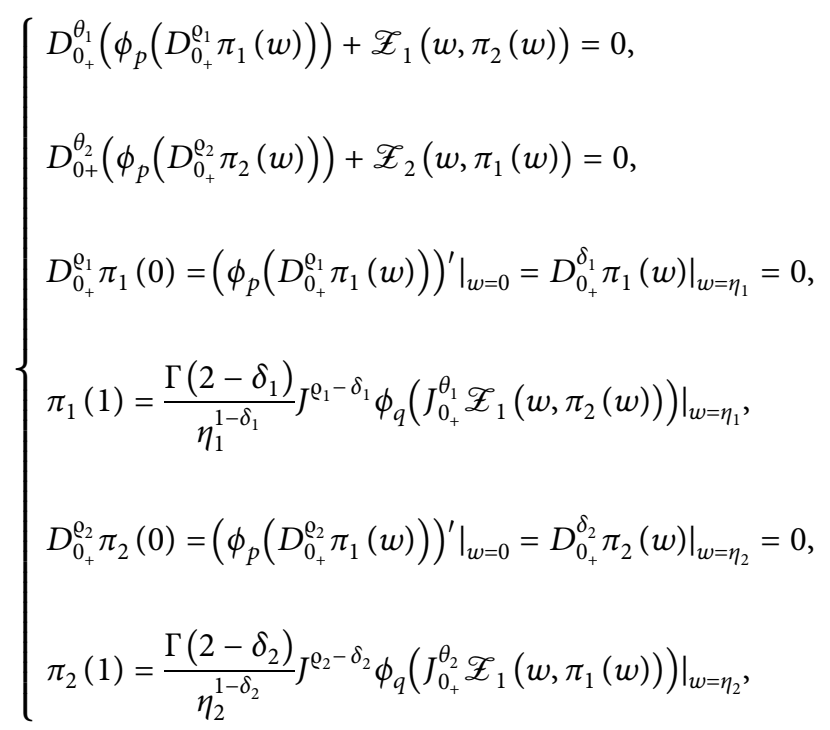

where $\varrho_{i}, \theta_{i} \in(1,2]$ and $\delta_{i}, \eta_{i} \in(0,1]$, for $i=1$ and 2 . The study of positive solutions to boundary value problems for fractional-order differential equations using the topological degree theory technique is rarely available in the literature, so this research field needs further elaboration. Most papers that dealt the topological degree theory with fractional orders belong to $(0,1)$ or $(1,2]$. For the uniqueness and existence analysis of nonlinear fractional differential equations, the case only Caputo fractional derivative is used frequently.

Thus, our motivation to this study is developing a sufficient condition for the coupled nonlinear fractional derivative that is based on both Caputo and Riemann-Liouville derivatives. The fractional order in our study is expanded to 
$(n-1, n]$, and we have used a technique of topological degree theory for the analysis of existence and uniqueness of our coupled system defined below. Besides, we have investigated Hyers-Ulam stability to the nonlinear coupled system of fractional-ordered ordinary differential equations with boundary conditions designed by the following:

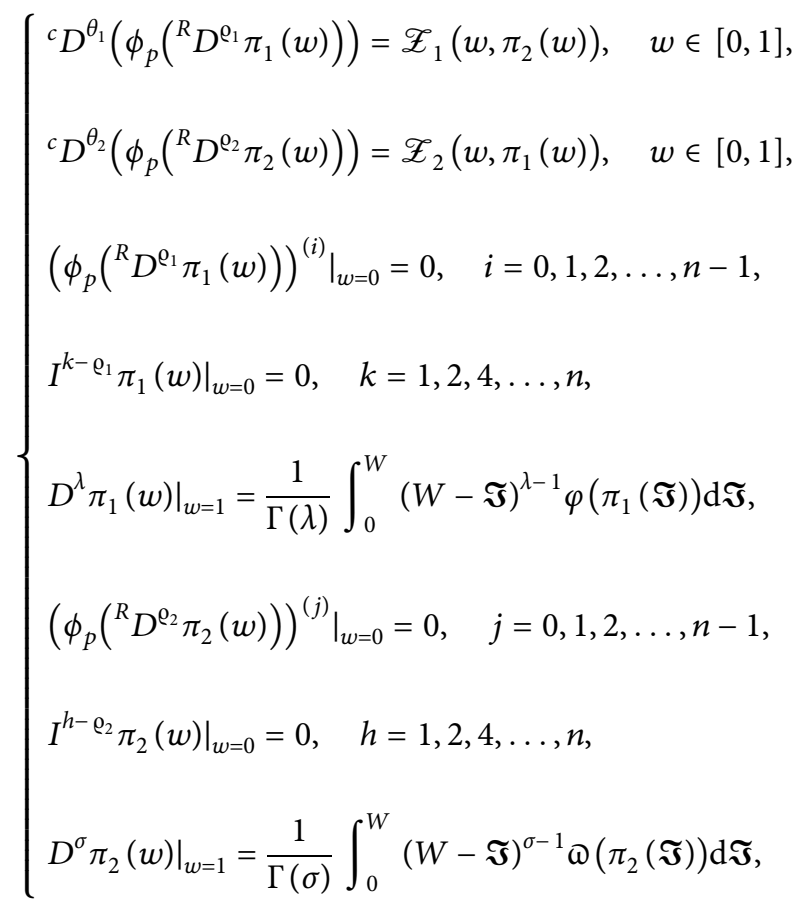

where $\varrho_{1}, \varrho_{2}, \theta_{1}, \theta_{2} \in(n-1, n], n \geq 3,1<\lambda, \sigma \leq 2,{ }^{c} D^{\theta_{1}}$ and ${ }^{c} D^{\theta_{2}}$ denote the Caputo fractional derivatives, ${ }^{R} D^{\mathrm{e}_{1}}$ and ${ }^{R} D^{\mathrm{e}_{2}}$ are the Riemann-Liouville fractional derivatives, and $\mathscr{Z}_{1}, \mathscr{Z}_{2}:[0,1] \times I R \longrightarrow I R$ are nonlinear functions, and the boundary functions $\varphi, \varpi \in L[0,1] . \phi_{p}$ represents the $p$-Laplacian operator such that $\phi_{p}(\nu)=\nu|\nu|^{p-2}$, and $\phi_{q}=$ $\phi_{p}^{-1}$ denotes the inverse of $p$-Laplacian, where $(1 / p)+(1 / q)=1$. Since it is difficult to find the exact solution of the nonlinear differential equations, stability and uniqueness have played a great role to get the approximate solution for the given nonlinear problems. Therefore, scientists and researchers have given attention to study the various forms of stability to the nonlinear problems in the sense of Ulam and their multiple types in the last few decades. We observe that the concept of Hyers-Ulam stability is fundamental in realistic problems, such as numerical analysis, biology, and economics (see [33-38]).

The remaining part of this manuscript is structured as follows. In Section 2, we have introduced some basic definitions and lemmas that we need to prove our main results. By using the topological degree theory, the results of existence and uniqueness for the solutions are obtained in Section 3. In Section 4, we investigate the stability of Hyers-Ulam to our proposed coupled system. The theoretical results are demonstrated by providing an example in Section 5, and finally, we have drawn the conclusion in Section 6.

\section{Preliminaries}

In this section, we introduce some basic notions, definitions, and important lemmas which are used in this article.Let $\Pi=$ $C([0,1], I R)$ be a Banach space for all continuous functions $\pi:[0,1] \longrightarrow I R$ with the norm $\|\pi\|=\sup \{|\pi(w)|: 0 \leq w \leq 1\}$. Further, $\Omega=\Pi \times \Pi$ is also a Banach space under the norms $\left\|\left(\pi_{1}, \pi_{2}\right)\right\|=\left\|\pi_{1}\right\|+\left\|\pi_{2}\right\|$ and $\left|\left(\pi_{1}, \pi_{2}\right)\right|=\max \left\{\left\|\pi_{1}\right\|,\left\|\pi_{2}\right\|\right\}$. The family of each bounded set of $P(\Omega)$ symbolized by $\mathfrak{B}$.

Definition 1. For $\mathscr{Z}(w):(0,+\infty) \longrightarrow \mathbb{R}$, the Caputo fractional derivative of noninteger order $\varrho>0$ is known by

$$
{ }^{c} D^{\varrho} \mathscr{Z}(w)=\frac{1}{\Gamma(n-\varrho)} \int_{0}^{w}(w-\mathfrak{\Im})^{n-\varrho-1} \mathscr{Z}^{(n)}(\mathfrak{J}) \mathrm{d} \mathfrak{\Im},
$$

where $n-1<\varrho<n$, the integral in the right side is pointwise defined on $(0, \infty)$, and $\mathscr{Z}(w)$ is a continuous function.

Definition 2. For $\mathscr{Z}(w):(0,+\infty) \longrightarrow \mathbb{R}$, the Riemann-Liouville fractional derivative of noninteger order $\varrho>0$ is known by

$$
{ }^{R} D^{\varrho} \mathscr{Z}(w)=\frac{1}{\Gamma(n-\varrho)}\left(\frac{\mathrm{d}}{\mathrm{d} w}\right)^{n} \int_{0}^{w}(w-\mathfrak{J})^{n-\varrho-1} \mathscr{Z}(\mathfrak{J}) \mathrm{d} \mathfrak{J},
$$

where $n-1<\varrho<n$, the integral in the right side is pointwise defined on $(0, \infty)$, and $\mathscr{Z}(w)$ is a continuous function.

Definition 3. For $\mathscr{Z}(w):(0,+\infty) \longrightarrow \mathbb{R}$, the Riemann-Liouville fractional integral of order $\varrho>0$ is defined by

$$
I^{\varrho} \mathscr{Z}(w)=\frac{1}{\Gamma(\varrho)} \int_{0}^{w}(w-\mathfrak{J})^{\varrho-1} \mathscr{Z}(\mathfrak{J}) \mathrm{d} \mathfrak{\Im},
$$

where the integral on the right side is pointwise defined on $(0,+\infty)$ and $\Gamma(\varrho)$ indicates the Gamma function defined as

$$
\Gamma(\varrho)=\int_{0}^{\infty} e^{-\mathfrak{I}} \mathfrak{J}^{\varrho^{-1}} \mathrm{~d} \mathfrak{\Im} .
$$

Lemma 1 (see [39]). Let $\mathrm{Q}>0$ and $\mathscr{Z} \in C(0,1) \cap L^{1}(0,1)$. Then, the general solution of the fractional differential equation $D^{\circ} \mathscr{Z}(w)=\pi(w)$ is given by

$$
\mathscr{Z}(w)=\pi(w)+c_{0}+c_{1} w+c_{2} w^{2}+\cdots+c_{n-1} w^{n-1},
$$

for $c_{i} \in \mathbb{R}, i=0,1,2, \ldots, n-1$.

Lemma 2 (see $[2,8])$. Let $\theta \in(n-1, n], \mathscr{Z} \in C^{n-1}$, and ${ }^{c} D^{\theta}$ is the fractional derivative for Caputo, then

$$
I^{\theta c} D^{\theta} \mathscr{Z}(w)=\mathscr{Z}(w)+a_{1}+a_{2} w+a_{3} w^{2}+a_{4} w^{3}+\cdots+a_{n} w^{n-1},
$$

for $a_{i} \in \mathbb{R}$ and $i=1,2,3,4, \ldots, n$.

Lemma 3 (see $[2,8])$. Let $\varrho \in(n-1, n], \mathscr{Z} \in C^{n-1}$, and ${ }^{R} D^{\varrho}$ is the fractional derivative for Riemman-Liouville, then 


$$
\begin{aligned}
I^{\varrho R} D^{\varrho} \mathscr{Z}(w)= & \mathscr{Z}(w)+b_{1} w^{\varrho-1}+b_{2} w^{\varrho-2}+b_{3} w^{\varrho-3} \\
& +a_{4} w^{\varrho-4}+\cdots+b_{n} w^{\varrho-n},
\end{aligned}
$$

for $b_{i} \in \mathbb{R}$ and $i=1,2,3,4, \ldots, n$.

Lemma 4 (see [22]). For $\varrho, \varepsilon>0$, the following relations are satisfying:

$$
\begin{aligned}
D^{\varrho} \xi^{\varepsilon} & =\frac{\Gamma(\varepsilon+1)}{\Gamma(1+\varepsilon-\varrho)} \xi^{\varepsilon-\varrho} \\
I^{\varrho} \xi^{\varepsilon} & =\frac{\Gamma(\varepsilon+1)}{\Gamma(1+\varepsilon+\varrho)} \xi^{\varepsilon+\varrho}
\end{aligned}
$$

Definition 4. The Kuratowski measure of noncompactness is the map $\vartheta: \mathfrak{B} \longrightarrow(0, \infty)$ known as $\vartheta(\mathscr{B})=\inf \{d>0: \mathscr{B}$ which admits a finite cover by sets of diameter $\leq d\}$, where $\mathscr{B} \in \mathfrak{B}$.

Proposition 1 (see [40]). The Kuratowski measure of $\vartheta$ satisfies the following properties:

(a) The Kuratowski measure $\vartheta(\mathscr{B})=0$; for a relative compact $\mathscr{B}$

(b) $\vartheta$ is a seminorm, i.e., $\vartheta(\mu \mathscr{B})=|\mu| \vartheta(\mathscr{B}), \mu \in I R$, and $\vartheta\left(\mathscr{B}_{1}+\mathscr{B}_{2}\right) \leq \vartheta\left(\mathscr{B}_{1}\right)+\vartheta\left(\mathscr{B}_{2}\right)$

(c) $\mathscr{B}_{1} \subset \mathscr{B}_{2}$ implies $\vartheta\left(\mathscr{B}_{1}\right) \leq \vartheta\left(\mathscr{B}_{2}\right) ; \vartheta\left(\mathscr{B}_{1} \cup \mathscr{B}_{2}\right)=$ $\sup \left\{\vartheta\left(\mathscr{B}_{1}\right), \vartheta\left(\mathscr{B}_{2}\right)\right\}$

(d) $\vartheta(\operatorname{conv} \mathscr{B})=\vartheta(\mathscr{B})$

(e) $\vartheta(\overline{\mathscr{B}})=\vartheta(\mathscr{B})$

Definition 5. Suppose that the function $\mathscr{F}: \Psi \longrightarrow \Pi$ is a continuous and bounded map, where $\Psi \subset \Pi$. $\mathscr{F}$ is called $\vartheta$-Lipschitz with $\eta \geq 0$, and if $\vartheta(\mathscr{F}(\mathscr{B})) \leq \eta \vartheta(\mathscr{B}), \forall \mathscr{B} \subset \Psi$ is bounded.

Moreover, if $\eta<0$, then $\mathscr{F}$ will be a strict $\vartheta$-contraction.

Definition 6. The function $\mathscr{F}$ is called 9 -condensing, and if $\vartheta(\mathscr{F}(\mathscr{B}))<\vartheta(\mathscr{B}), \forall \mathscr{B} \subset \Psi$ is bounded with $\vartheta(\mathscr{B})>0$.

In other words, $\vartheta(\mathscr{F}(\mathscr{B})) \geq \vartheta(\mathscr{B})$ implies $\vartheta(\mathscr{B})=0$.

We indicate that the class of each $\vartheta$-condensing mappings $\mathscr{F}: \Psi \longrightarrow \Pi$ by $C_{\vartheta}(\Psi)$ and the class of each strict $\vartheta$-contractions $\mathscr{F}: \Psi \longrightarrow \Pi$ by $\zeta C_{\vartheta}(\Psi)$.

We remark that $C_{\vartheta}(\Psi)^{\zeta C_{\vartheta}(\Psi)}$, and every $\mathscr{F} \in C_{9}(\Psi)$ is $\vartheta$-Lipschitz with $\eta=1$. As well, we recall that $\mathscr{F}: \Psi \longrightarrow \Pi$ is Lipschitz if $\exists \eta>0$ such that $\|\mathscr{F}(\pi)-\mathscr{F}(\bar{\pi})\| \leq \eta\|\pi-\bar{\pi}\|$, and $\forall \pi, \bar{\pi} \in \Psi$. Also, $\mathscr{F}$ is a strict contraction under the condition $\eta<1$.

Proposition 2 (see [31]). Let $\mathscr{F}, \mathscr{G}: \Psi \longrightarrow \Pi$ be 9 -Lipschitz operators with constants $\eta_{1}$ and $\eta_{2}$, respectively, then $\mathscr{F}+$ $\mathscr{G}: \Psi \longrightarrow \Pi$ is $\vartheta$-Lipschitz with constants $\eta_{1}+\eta_{2}$.

Proposition 3 (see [41]). The operator $\mathscr{F}: \Psi \longrightarrow \Pi$ is compact if and only if $\mathscr{F}$ is 9 -Lipschitz with $\eta=0$.
Proposition 4 (see [31]). The operator $\mathscr{F}: \Psi \longrightarrow \Pi$ is Lipschitz with constant $\eta$ if and only if $\mathscr{F}$ is 9 -Lipschitz with constant $\eta$.

Lemma 5 (see [39]). Let $\phi_{p}$ be a nonlinear p-Laplacian operator.

(1) If $1<p \leq 2, j_{1} j_{2}>0$, and $\left|j_{1}\right|,\left|j_{2}\right| \geq \rho>0$, then

$$
\left|\phi_{p}\left(j_{1}\right)-\phi_{p}\left(j_{2}\right)\right| \leq(p-1) \rho^{p-2}\left|j_{1}-j_{2}\right|
$$

(2) If $p>2$ and $\left|j_{1}\right|,\left|j_{2}\right| \leq \rho^{*}$, then

$$
\left|\phi_{p}\left(j_{1}\right)-\phi_{p}\left(j_{2}\right)\right| \leq(p-1) \rho^{* p-2}\left|j_{1}-j_{2}\right| .
$$

Theorem 1 (see [29]). Let $\mathscr{F}: \Omega \longrightarrow \Omega$ be a 9 -contraction, and $\Xi=\{\omega \in \Omega: \exists, 0 \leq \rho \leq 1$ such that $\omega=\rho \mathscr{F} \omega$. If $\Xi \subset \Omega$ is $a$ bounded set, there exists $r>0$ such that $\Xi \subset \mathscr{B}_{r}(0)$, then the degree $\operatorname{deg}\left(I-\rho \mathscr{F}, \mathscr{B}_{r}(0), 0\right)=1, \forall \rho \in[0,1]$.

Thus, $\mathscr{F}$ has at least one fixed point, and the set of the fixed points of $\mathscr{F}$ lies in $\mathscr{B}_{r}(0)$.

The above theorem that we mentioned plays a substantial role in obtaining our main results.

\section{Main Results}

In the current section, we establish some appropriate conditions for proposed coupled system (5).

Theorem 2. Let $\mathscr{Z}:[0,1] \longrightarrow I R$ be a $\varrho_{1}$ times' integrable function. Then, for $\varrho_{1} \in(3, n]$ and positive integer $n \geq 4$, the solution of the boundary value problem is as follows:

$$
\left\{\begin{array}{l}
{ }^{c} D^{\theta_{1}}\left(\phi_{p}\left({ }^{R} D^{\varrho_{1}} \pi_{1}(w)\right)\right)=\mathscr{Z}_{1}\left(w, \pi_{2}(w)\right), \quad w \in[0,1], \\
\left.\left(\phi_{p}\left({ }^{R} D^{\varrho_{1}} \pi_{1}(w)\right)\right)^{(i)}\right|_{w=0}=0, \quad i=0,1,2, \ldots, n-1, \\
\left.I^{k-\varrho_{1}} \pi_{1}(w)\right|_{w=0}=0, \quad k=1,2,4, \ldots, n, \\
\left.D^{\lambda} \pi_{1}(w)\right|_{w=1}=\frac{1}{\Gamma(\lambda)} \int_{0}^{W}(W-\mathfrak{J})^{\lambda-1} \varphi\left(\pi_{1}(\mathfrak{J})\right) \mathrm{d} \mathfrak{J}
\end{array}\right.
$$

is given by

$$
\begin{aligned}
\pi_{1}(w)= & \frac{\Gamma\left(\varrho_{1}-\lambda-2\right) w^{\varrho_{1}-3}}{\Gamma\left(\varrho_{1}-2\right) \Gamma(\lambda)} \int_{0}^{W}(W-\mathfrak{I})^{\lambda-1} \varphi\left(\pi_{1}(\mathfrak{I})\right) \mathrm{d} \mathfrak{I} \\
& +\int_{0}^{1} G^{\varrho_{1}}(w, \mathfrak{I}) \phi_{q}\left(\frac{1}{\Gamma\left(\theta_{1}\right)} \int_{0}^{\Im}(\mathfrak{I}-\tau)^{\theta_{1}-1} \mathscr{Z}_{1}\left(\tau, \pi_{2}(\tau)\right) \mathrm{d} \tau\right) \mathrm{d} \mathfrak{J},
\end{aligned}
$$

where $G^{\varrho_{1}}(w, \mathfrak{\Im})$ is the Green's function provided by 


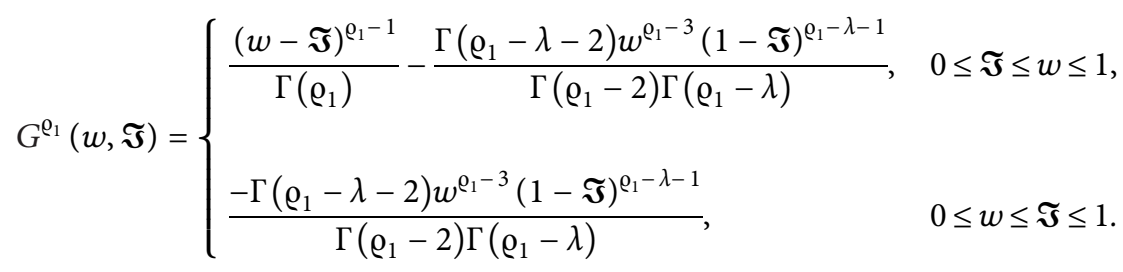

Proof. Applying the integral operator $I^{\theta_{1}}$ and using Lemma 2 on (16), we get

$$
\begin{aligned}
\phi_{p}\left[{ }^{R} D^{\varrho_{1}} \pi_{1}(w)\right]= & I^{\theta_{1}} \mathscr{Z}_{1}\left(w, \pi_{2}(w)\right)+a_{1}+a_{2} w \\
& +a_{3} w^{2}+a_{4} w^{3}+\cdots+a_{n} w^{n-1} .
\end{aligned}
$$

Using the condition $\left.\left(\phi_{p}\left[{ }^{R} D^{\varrho_{1}} \pi_{1}(w)\right]\right)^{(i)}\right|_{w=0}=0$, for $i=$ $0,1,2, \ldots, n-1$, in (19), we obtain $a_{1}=a_{2}=a_{3}=$ $\cdots=a_{n}=0$, and then, we get

$$
\phi_{p}\left[{ }^{R} D^{\varrho_{1}} \pi_{1}(w)\right]=I^{\theta_{1}} \mathscr{Z}_{1}\left(w, \pi_{2}(w)\right) .
$$

From (20), we have

$$
{ }^{R} D^{\varrho_{1}} \pi_{1}(w)=\phi_{q}\left(I^{\theta_{1}}\left(\mathscr{Z}_{1}\left(w, \pi_{2}(w)\right)\right) .\right.
$$

Applying the operator $I^{Q_{1}}$ and using Lemma 3 in (21), we get

$$
\begin{aligned}
\pi_{1}(w)= & I^{\varrho_{1}}\left(\phi_{q}\left(I^{\theta_{1}}\left(\mathscr{Z}_{1}\left(w, \pi_{2}(w)\right)\right)\right)\right)+b_{1} w^{\varrho_{1}-1} \\
& +b_{2} w^{\varrho_{1}-2}+b_{3} w^{\varrho_{1}-3}+b_{4} w^{\varrho^{-4}}+\cdots+b_{n} w^{\varrho_{1}-n} .
\end{aligned}
$$

Using the condition $\left.I^{k-\varrho_{1}} \pi(w)\right|_{w=0}=0, k=1,2,4, \ldots, n$, we get $b_{1}=b_{2}=b_{4}=\ldots=b_{n}=0$, and then, we obtain

$$
\pi_{1}(w)=I^{\varrho_{1}}\left(\phi_{q}\left(I^{\theta_{1}}\left(\mathscr{Z}_{1}\left(w, \pi_{2}(w)\right)\right)\right)\right)+b_{3} w^{\varrho_{1}-3} .
$$

Using the condition $\left.D^{\lambda} \pi_{1}(w)\right|_{w=1}={ }_{0} I_{W}^{\lambda} \varphi\left(\pi_{1}\right)=$ $(1 / \Gamma(\lambda)) \int_{0}^{W}(W-\mathfrak{J})^{\lambda-1} \varphi\left(\pi_{1}(\mathfrak{I})\right) \mathrm{d} \mathfrak{\Im}$ and Lemma 4 in $(22)$, we get

$$
b_{3}=\frac{\Gamma\left(\varrho_{1}-\lambda-2\right)}{\Gamma\left(\varrho_{1}-2\right)}\left[{ }_{0} I_{W}^{\lambda} \varphi\left(\pi_{1}\right)-I^{\varrho_{1}-\lambda}\left(\phi_{q}\left(I^{\theta_{1}}\left(\mathscr{Z}_{1}\left(1, \pi_{2}(1)\right)\right)\right)\right)\right] .
$$

Putting the value of $b_{3}$ in (23), we get

$$
\begin{aligned}
\pi_{1}(w)= & \frac{1}{\Gamma\left(\varrho_{1}\right)} \int_{0}^{w}(w-\mathfrak{J})^{\varrho_{1}-1} \phi_{q}\left(\frac{1}{\Gamma\left(\theta_{1}\right)} \int_{0}^{\mathfrak{J}}(\mathfrak{I}-\tau)^{\theta_{1}-1} \mathscr{Z}_{1}\left(\left(\tau, \pi_{2}(\tau)\right) \mathrm{d} \tau\right)\right) \mathrm{d} \mathfrak{I} \\
& +\frac{\Gamma\left(\varrho_{1}-\lambda-2\right) w^{\varrho_{1}-3}}{\Gamma\left(\varrho_{1}-2\right) \Gamma(\lambda)} \int_{0}^{W}(W-\mathfrak{J})^{\lambda-1} \varphi\left(\pi_{1}(\mathfrak{J})\right) \mathrm{d} \mathfrak{J} \\
& -\frac{\Gamma\left(\varrho_{1}-\lambda-2\right) w^{\varrho_{1}-3}}{\Gamma\left(\varrho_{1}-2\right) \Gamma\left(\varrho_{1}-\lambda\right)} \int_{0}^{1}(1-\mathfrak{I})^{\varrho_{1}-\lambda-1} \phi_{q}\left(\frac{1}{\Gamma\left(\theta_{1}\right)} \int_{0}^{\mathfrak{J}}(\mathfrak{I}-\tau)^{\theta_{1}-1} \mathscr{Z}_{1}\left(\tau, \pi_{2}(\tau)\right) \mathrm{d} \tau\right) \mathrm{d} \mathfrak{I}
\end{aligned}
$$

which can be written after rearranging as follows:

$$
\begin{aligned}
& \pi_{1}(w)=\frac{\Gamma\left(\varrho_{1}-\lambda-2\right) w^{\varrho_{1}-3}}{\Gamma\left(\varrho_{1}-2\right) \Gamma(\lambda)} \int_{0}^{W}(W-\mathfrak{J})^{\lambda-1} \varphi\left(\pi_{1}(\mathfrak{\Im})\right) \mathrm{d} \mathfrak{\Im} \\
& +\int_{0}^{1} G^{\mathrm{e}_{1}}(w, \mathfrak{J}) \phi_{q}\left(\frac{1}{\Gamma\left(\theta_{1}\right)} \int_{0}^{\mathfrak{J}}(\mathfrak{I}-\tau)^{\theta_{1}-1} \mathscr{Z}_{1}\left(\left(\tau, \pi_{2}(\tau)\right) \mathrm{d} \tau\right)\right) \mathrm{d} \mathfrak{J},
\end{aligned}
$$

where $G^{\varrho_{1}}(w, \mathfrak{I})$ is the Green's function defined in (18).

In view of Theorem 2, the identical coupled system of Hammerstein-kind integral equations to fractional differential equation coupled system (5) is given as follows:

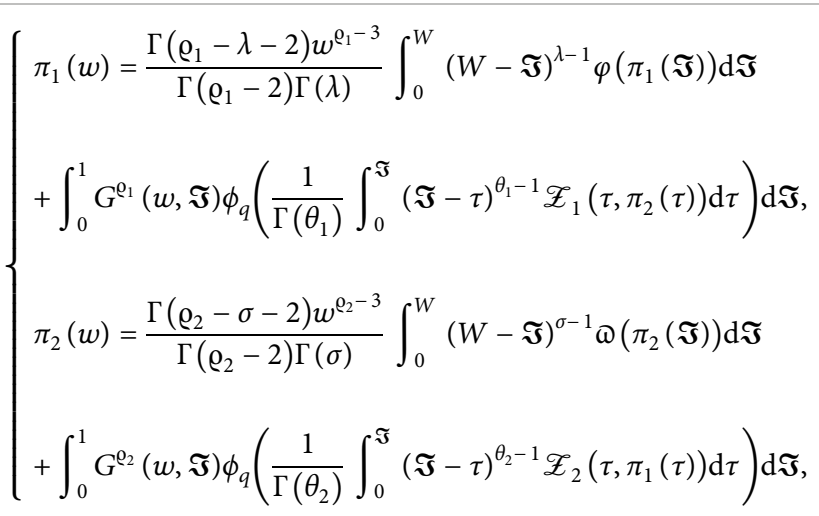

where $G^{\varrho_{2}}(w, \mathfrak{I})$ is the Green's function provided by 


$$
G^{\varrho_{2}}(w, \mathfrak{J})= \begin{cases}\frac{(w-\mathfrak{J})^{\varrho_{2}-1}}{\Gamma\left(\varrho_{2}\right)}-\frac{\Gamma\left(\varrho_{2}-\sigma-2\right) w^{\varrho_{2}-3}(1-\mathfrak{J})^{\varrho_{2}-\sigma-1}}{\Gamma\left(\varrho_{2}-2\right) \Gamma\left(\varrho_{2}-\sigma\right)}, & 0 \leq \mathfrak{J} \leq w \leq 1, \\ \frac{-\Gamma\left(\varrho_{2}-\sigma-2\right) w^{\varrho_{2}-3}(1-\mathfrak{J})^{\varrho_{2}-\sigma-1}}{\Gamma\left(\varrho_{2}-2\right) \Gamma\left(\varrho_{2}-\sigma\right)}, & 0 \leq w \leq \mathfrak{J} \leq 1 .\end{cases}
$$

From $G^{\mathrm{Q}_{1}}(w, \mathfrak{\Im})$ and $G^{\mathrm{Q}_{2}}(w, \mathfrak{\Im})$ obviously,

$$
\begin{gathered}
\mathscr{F}_{1}\left(\pi_{1}\right)(w)=\frac{\Gamma\left(\varrho_{1}-\lambda-2\right) w^{\varrho_{1}-3}}{\Gamma\left(\varrho_{1}-2\right) \Gamma(\lambda)} \int_{0}^{W}(W-\mathfrak{J})^{\lambda-1} \varphi\left(\pi_{1}(\mathfrak{I})\right) \mathrm{d} \mathfrak{I}, \\
\mathscr{F}_{2}\left(\pi_{2}\right)(w)=\frac{\Gamma\left(\varrho_{2}-\sigma-2\right) w^{\varrho_{2}-3}}{\Gamma\left(\varrho_{2}-2\right) \Gamma(\sigma)} \int_{0}^{W}(W-\mathfrak{J})^{\sigma-1} \varpi\left(\pi_{2}(\mathfrak{I})\right) \mathrm{d} \mathfrak{I}, \\
\mathscr{G}_{1}, \mathscr{G}_{2}: \Omega \longrightarrow \Omega,
\end{gathered}
$$$$
\max _{w \in[0,1]}\left|G^{\varrho_{1}}(w, \mathfrak{\Im})\right|=\frac{\Gamma\left(\varrho_{1}-\lambda-2\right)(1-\mathfrak{\Im})^{\varrho_{1}-\lambda-1}}{\Gamma\left(\varrho_{1}-2\right) \Gamma\left(\varrho_{1}-\lambda\right)},
$$$$
\max _{w \in[0,1]}\left|G^{\varrho_{2}}(w, \mathfrak{J})\right|=\frac{\Gamma\left(\varrho_{2}-\sigma-2\right)(1-\mathfrak{J})^{\varrho_{2}-\sigma-1}}{\Gamma\left(\varrho_{2}-2\right) \Gamma\left(\varrho_{2}-\sigma\right)}, \quad \mathfrak{I} \in[0,1] .
$$

We define the operators $\mathscr{F}_{1}: \Pi_{1} \longrightarrow \Pi_{1}$ and $\mathscr{F}_{2}$ :

as $\Pi_{2} \longrightarrow \Pi_{2}$ as

$$
\begin{aligned}
& \mathscr{G}_{1}\left(\pi_{2}\right)(w)=\int_{0}^{1} G^{\varrho_{1}}(w, \mathfrak{I}) \phi_{q}\left(\frac{1}{\Gamma\left(\theta_{1}\right)} \int_{0}^{\mathfrak{I}}(\mathfrak{I}-\tau)^{\theta_{1}-1} \mathscr{Z}_{1}\left(\tau, \pi_{2}(\tau)\right) \mathrm{d} \tau\right) \mathrm{d} \mathfrak{I}, \\
& \mathscr{G}_{2}\left(\pi_{1}\right)(w)=\int_{0}^{1} G^{\varrho_{2}}(w, \mathfrak{J}) \phi_{q}\left(\frac{1}{\Gamma\left(\theta_{2}\right)} \int_{0}^{\mathfrak{I}}(\mathfrak{I}-\tau)^{\theta_{2}-1} \mathscr{Z}_{2}\left(\tau, \pi_{1}(\tau)\right) \mathrm{d} \tau\right) \mathrm{d} \mathfrak{I} .
\end{aligned}
$$

Therefore, we have $\mathscr{F}\left(\pi_{1}, \pi_{2}\right)=\left(\mathscr{F}_{1}, \mathscr{F}_{2}\right)\left(\pi_{1}\right.$, $\left.\pi_{2}\right), \mathscr{G}\left(\pi_{1}, \quad \pi_{2}\right)=\left(\mathscr{G}_{1}, \mathscr{G}_{2}\right)\left(\pi_{1}, \pi_{2}\right), \quad$ and $\mathscr{T}\left(\pi_{1}, \pi_{2}\right)=$ $\mathscr{F}\left(\pi_{1}, \pi_{2}\right)+\mathscr{G}\left(\pi_{1}, \pi_{2}\right)$. Thus, the equivalent operator equation for the toppled system of Hammerstein-kind integral equations (27) is provided by

$$
\left(\pi_{1}, \pi_{2}\right)=\mathscr{T}\left(\pi_{1}, \pi_{2}\right)=\mathscr{F}\left(\pi_{1}, \pi_{2}\right)+\mathscr{G}\left(\pi_{1}, \pi_{2}\right) .
$$

Consequently, the solutions of system (27) are the fixed points of operator equation (32).

Now, we need to list the following assumptions to complete our results.

$\left(H_{1}\right)$ For $\hbar, \pi_{1}, \ell, \pi_{2} \in I R$, the nonlocal functions $\varphi$ and $\Phi$ satisfy $\left\|\varphi(\hbar)-\varphi\left(\pi_{1}\right)\right\| \leq K_{\varphi}\left\|\hbar-\pi_{1}\right\|$ and $\| \omega$ $(\ell)-\Phi\left(\pi_{2}\right)\left\|\leq K_{\omega}\right\| \ell-\pi_{2} \|$ such that $K_{\varphi}, K_{\oplus} \in[0,1)$

$\left(\mathrm{H}_{2}\right) \quad$ With the positive constants given $C_{\varphi}, C_{\oplus}, N_{\varphi}, N_{\oplus}$, and $q_{1} \in[0,1)$, the nonlocal functions $\varphi$ and $\omega$ for $\pi_{1}, \pi_{2} \in I R$ satisfy the following growth conditions $\left|\varphi\left(\pi_{1}\right)\right| \leq C_{\varphi}\left|\pi_{1}\right|^{q_{1}}+N_{\varphi}$ and $\left|\omega\left(\pi_{2}\right)\right| \leq C_{\oplus}$ $\left|\pi_{2}\right|^{q_{1}}+N_{\oplus}$
$\left(H_{3}\right)$ With the presence of constants $g, h, N_{\mathscr{Z}_{1}}, N_{\mathscr{I}_{2}}$, and $q_{2} \in[0,1)$, the nonlinear functions $\mathscr{Z}_{1}$ and $\mathscr{E}_{2}$ for $\pi_{1}, \pi_{2} \in I R$ satisfy the following growth conditions:

$$
\begin{aligned}
& \left|\mathscr{Z}_{1}\left(w, \pi_{2}\right)\right| \leq \phi_{p}\left(g\left|\pi_{2}\right|^{q_{2}}+N_{\mathscr{E}_{1}}\right), \\
& \left|\mathscr{Z}_{2}\left(w, \pi_{1}\right)\right| \leq \phi_{p}\left(h\left|\pi_{1}\right|^{q_{2}}+N_{\mathscr{Z}_{2}}\right) .
\end{aligned}
$$

$\left(H_{4}\right)$ For $\hbar, \pi_{1}, \ell, \pi_{2} \in I R$, there exists positive constants $L_{\mathscr{E}_{1}}$ and $L_{\mathscr{E}_{2}}$ such that

$$
\begin{gathered}
\left|\mathscr{Z}_{1}(w, \ell)-\mathscr{Z}_{1}\left(w, \pi_{2}\right)\right| \leq L_{\mathscr{Z}_{1}}\left|\ell-\pi_{2}\right|, \\
\left|\mathscr{Z}_{2}(w, \hbar)-\mathscr{Z}_{2}\left(w, \pi_{1}\right)\right| \leq L_{\mathscr{Z}_{2}}\left|\hbar-\pi_{1}\right| .
\end{gathered}
$$

Theorem 3. Assume that $\left(H_{1}\right)$ and $\left(H_{2}\right)$ hold true. Then, the operator $\mathscr{F}$ is Lipschitz and satisfies the following growth condition:

$$
\left\|\mathscr{F}\left(\pi_{1}, \pi_{2}\right)\right\| \leq C_{\mathscr{F}}\left\|\left(\pi_{1}, \pi_{2}\right)\right\|^{q_{1}}+N_{\mathscr{F}}, \quad \forall\left(\pi_{1}, \pi_{2}\right) \in \Omega .
$$


Proof. By assumption $\left(H_{1}\right)$, we get

$$
\begin{aligned}
\left|\mathscr{F}_{1}\left(\pi_{1}\right)(w)-\mathscr{F}_{1}\left(\overline{\pi_{1}}\right)(w)\right| & =\left|\frac{\Gamma\left(\varrho_{1}-\lambda-2\right)}{\Gamma\left(\varrho_{1}-2\right) \Gamma(\lambda)} \int_{0}^{W}(W-\Im)^{\lambda-1}\left[\varphi\left(\pi_{1}\right)-\varphi\left(\overline{\pi_{1}}\right)\right] \mathrm{d} \Im\right| \\
& \leq \frac{\Gamma\left(\varrho_{1}-\lambda-2\right)}{\Gamma\left(\varrho_{1}-2\right) \Gamma(\lambda)} \int_{0}^{W}(W-\mathfrak{\Im})^{\lambda-1}\left|\varphi\left(\pi_{1}\right)-\varphi\left(\overline{\pi_{1}}\right)\right| \mathrm{d} \Im
\end{aligned}
$$

which yields

$$
\left\|\mathscr{F}_{1}\left(\pi_{1}\right)-\mathscr{F}_{1}\left(\overline{\pi_{1}}\right)\right\| \leq \bar{K}_{\varphi}\left\|\pi_{1}-\overline{\pi_{1}}\right\|,
$$

where

$$
\bar{K}_{\varphi}=\frac{K_{\varphi} \Gamma\left(\varrho_{1}-\lambda-2\right) W^{\lambda}}{\Gamma\left(\varrho_{1}-2\right) \Gamma(\lambda+1)} \in[0,1) .
$$

To get the growth condition, consider

$$
\begin{aligned}
\left|\mathscr{F}_{1}\left(\pi_{1}\right)(w)\right|= & \left|\frac{\Gamma\left(\varrho_{1}-\lambda-2\right)}{\Gamma\left(\varrho_{1}-2\right) \Gamma(\lambda)} \int_{0}^{W}(W-\Im)^{\lambda-1} \varphi\left(\pi_{1}(\Im)\right) \mathrm{d} \Im\right| \\
& \leq \frac{\Gamma\left(\varrho_{1}-\lambda-2\right)}{\Gamma\left(\varrho_{1}-2\right) \Gamma(\lambda)} \int_{0}^{W}(W-\Im)^{\lambda-1}\left|\varphi\left(\pi_{1}(\mathfrak{\Im})\right)\right| \mathrm{d} \mathfrak{J},
\end{aligned}
$$

$$
\left\|\mathscr{F}_{1} \pi_{1}\right\|_{c} \leq \frac{\Gamma\left(\varrho_{1}-\lambda-2\right) W^{\lambda}}{\Gamma\left(\varrho_{1}-2\right) \Gamma(\lambda+1)}\left[C_{\varphi}\left\|\pi_{1}\right\|_{c}^{q_{1}}+N_{\varphi}\right] .
$$

In a similar manner, we have

$$
\begin{aligned}
& \left|\mathscr{F}_{2}\left(\pi_{2}\right)(w)\right|=\left|\frac{\Gamma\left(\varrho_{2}-\sigma-2\right)}{\Gamma\left(\varrho_{2}-2\right) \Gamma(\sigma)} \int_{0}^{W}(W-\mathfrak{J})^{\sigma-1} 凹\left(\pi_{2}(\mathfrak{\Im})\right) \mathrm{d} \Im\right| \\
& \leq \frac{\Gamma\left(\varrho_{2}-\sigma-2\right)}{\Gamma\left(\varrho_{2}-2\right) \Gamma(\sigma)} \int_{0}^{W}(W-\mathfrak{\Im})^{\sigma-1}\left|\bowtie\left(\pi_{2}(\mathfrak{I})\right)\right| \mathrm{d} \mathfrak{I}
\end{aligned}
$$

which implies that

$$
\left\|\mathscr{F}_{2} \pi_{2}\right\|_{c} \leq \frac{\Gamma\left(\varrho_{2}-\sigma-2\right) W^{\sigma}}{\Gamma\left(\varrho_{2}-2\right) \Gamma(\sigma+1)}\left[C_{\omega}\left\|\pi_{2}\right\|_{c}^{q_{1}}+N_{\varpi}\right] .
$$

Now,

which means that

$$
\begin{aligned}
& \left\|\mathscr{F}\left(\pi_{1}, \pi_{2}\right)\right\|_{c} \leq \frac{\Gamma\left(\varrho_{1}-\lambda-2\right) W^{\lambda}}{\Gamma\left(\varrho_{1}-2\right) \Gamma(\lambda+1)}\left[C_{\varphi}\left\|\pi_{1}\right\|_{c}^{q_{1}}+N_{\varphi}\right] \\
& +\frac{\Gamma\left(\varrho_{2}-\sigma-2\right) W^{\sigma}}{\Gamma\left(\varrho_{2}-2\right) \Gamma(\sigma+1)}\left[C_{\omega}\left\|\pi_{2}\right\|_{c}^{q_{1}}+N_{\varpi}\right] \\
& \leq\left(\frac{\Gamma\left(\varrho_{1}-\lambda-2\right) W^{\lambda}}{\Gamma\left(\varrho_{1}-2\right) \Gamma(\lambda+1)} C_{\varphi}\left\|\pi_{1}\right\|_{c}^{q_{1}}\right. \\
& \left.+\frac{\Gamma\left(\varrho_{2}-\sigma-2\right) W^{\sigma}}{\Gamma\left(\varrho_{2}-2\right) \Gamma(\sigma+1)} C_{\omega}\left\|\pi_{2}\right\|_{c}^{q_{1}}\right) \\
& +\left(\frac{\Gamma\left(\varrho_{1}-\lambda-2\right) W^{\lambda} N_{\varphi}}{\Gamma\left(\varrho_{1}-2\right) \Gamma(\lambda+1)}+\frac{\Gamma\left(\varrho_{2}-\sigma-2\right) W^{\sigma} N_{\emptyset}}{\Gamma\left(\varrho_{2}-2\right) \Gamma(\sigma+1)}\right) . \\
& C_{\mathscr{F}}=\max \left\{\frac{\Gamma\left(\varrho_{1}-\lambda-2\right) W^{\lambda}}{\Gamma\left(\varrho_{1}-2\right) \Gamma(\lambda+1)} C_{\varphi}, \frac{\Gamma\left(\varrho_{2}-\sigma-2\right) W^{\sigma}}{\Gamma\left(\varrho_{2}-2\right) \Gamma(\sigma+1)} C_{\emptyset}\right\}, \\
& \left\|\mathscr{F}\left(\pi_{1}, \pi_{2}\right)\right\|_{\mathcal{C}} \leq C_{\mathscr{F}}\left[\left\|\pi_{1}\right\|^{q_{1}}+\left\|\pi_{2}\right\|^{q_{1}}\right]+N_{\mathscr{F}}
\end{aligned}
$$

Thus,$$
=C_{\mathscr{F}}\left\|\left(\pi_{1}, \pi_{2}\right)\right\|^{q_{1}}+N_{\mathscr{F}} \text {, }
$$

where 
Theorem 4. Suppose that $\left(\mathrm{H}_{3}\right)$ is satisfied. Then, the operator $\mathscr{G}$ is continuous and satisfies the following growth condition:

$$
\left\|G\left(\pi_{1}, \pi_{2}\right)\right\| \leq \Lambda\left\|\left(\pi_{1}, \pi_{2}\right)\right\|^{q_{2}}+\Theta, \quad \text { for all }\left(\pi_{1}, \pi_{2}\right) \in \Omega,
$$

where $\Lambda=\gamma(g+h)$ and $\Theta=\gamma\left(N_{\mathscr{I}_{1}}+N_{\mathscr{Z}_{2}}\right)$ such that

$$
\begin{aligned}
\gamma=\max \{ & \left(\frac{1}{\Gamma\left(\varrho_{1}+1\right)}+\frac{\Gamma\left(\varrho_{1}-\lambda-2\right)}{\Gamma\left(\varrho_{1}-2\right) \Gamma\left(\varrho_{1}-\lambda+1\right)}\right)\left[\frac{1}{\Gamma\left(\theta_{1}+1\right)}\right]^{q-1}, \\
& \left.\cdot\left(\frac{1}{\Gamma\left(\varrho_{2}+1\right)}+\frac{\Gamma\left(\varrho_{2}-\sigma-2\right)}{\Gamma\left(\varrho_{2}-2\right) \Gamma\left(\varrho_{2}-\sigma+1\right)}\right)\left[\frac{1}{\Gamma\left(\theta_{2}+1\right)}\right]^{q-1}\right\} .
\end{aligned}
$$

Proof. Let $\mathfrak{B}_{r}=\left\{\left(\pi_{1}, \pi_{2}\right) \in \Omega:\left\|\left(\pi_{1}, \pi_{2}\right)\right\| \leq r\right\}$ be a bounded set with a sequence $\left(\pi_{1_{n}}, \pi_{2_{n}}\right)$ converging to $\left(\pi_{1}, \pi_{2}\right)$ in $\mathfrak{B}_{r}$. In order to show that $\mathscr{G}$ is continuous, we have to prove that
$\left\|\mathscr{G}\left(\pi_{1_{n}}, \pi_{2_{n}}\right)-\mathscr{G}\left(\pi_{1}, \pi_{2}\right)\right\| \longrightarrow 0$ as $n \longrightarrow \infty$. Let us choose the following:

$$
\begin{aligned}
& \left|\left(\mathscr{G}_{1}\left(\pi_{2_{n}}\right)-\mathscr{G}_{1}\left(\pi_{2}\right)\right)(w)\right| \\
& =\mid \int_{0}^{1} G^{\varrho_{1}}(w, \mathfrak{I}) \phi_{q}\left(\frac{1}{\Gamma\left(\theta_{1}\right)} \int_{0}^{\mathfrak{I}}(\mathfrak{I}-\tau)^{\theta_{1}-1} \mathscr{Z}_{1}\left(\tau, \pi_{2_{n}}(\tau)\right) \mathrm{d} \tau\right) \mathrm{d} \mathfrak{I} \\
& -\int_{0}^{1} G^{\varrho_{1}}(w, \mathfrak{I}) \phi_{q}\left(\frac{1}{\Gamma\left(\theta_{1}\right)} \int_{0}^{\mathfrak{I}}(\mathfrak{I}-\tau)^{\theta_{1}-1} \mathscr{Z}_{1}\left(\tau, \pi_{2}(\tau) \mathrm{d} \tau\right) \mathrm{d} \mathfrak{I}\right) \mid \\
& \leq \int_{0}^{1}\left\|G^{\varrho_{1}}(w, \mathfrak{I})\right\| \phi_{q}\left[\frac{1}{\Gamma\left(\theta_{1}\right)} \int_{0}^{\mathfrak{I}}(\mathfrak{I}-\tau)^{\theta_{1}-1} \mathscr{Z}_{1}\left(\tau, \pi_{2_{n}}(\tau) \mathrm{d} \tau\right)\right] \\
& -\phi_{q}\left[\frac{1}{\Gamma\left(\theta_{1}\right)} \int_{0}^{\mathfrak{J}}(\mathfrak{I}-\tau)^{\theta_{1}-1} \mathscr{Z}_{1}\left(\tau, \pi_{2}(\tau) \mathrm{d} \tau\right)\right] \mid \mathrm{d} \mathfrak{I} \\
& \leq(q-1) \rho_{1}^{q-2} \int_{0}^{1}\left\|G^{\varrho_{1}}(w, \mathfrak{J})\right\| \frac{1}{\Gamma\left(\theta_{1}\right)} \int_{0}^{\mathfrak{J}}(\mathfrak{J}-\tau)^{\theta_{1}-1} \mathscr{Z}_{1}\left(\tau, \pi_{2_{n}}(\tau) \mathrm{d} \tau\right) \\
& -\frac{1}{\Gamma\left(\theta_{1}\right)} \int_{0}^{\mathfrak{J}}(\mathfrak{I}-\tau)^{\theta_{1}-1} \mathscr{Z}_{1}\left(\tau, \pi_{2}(\tau) \mathrm{d} \tau\right) \mid \mathrm{d} \mathfrak{I} \\
& \leq(q-1) \rho_{1}^{q-2} \int_{0}^{1}\left|G^{\varrho_{1}}(w, \mathfrak{J})\right|\left[\frac{1}{\Gamma\left(\theta_{1}\right)} \int_{0}^{\mathfrak{J}}(\mathfrak{I}-\tau)^{\theta_{1}-1}\left|\mathscr{Z}_{1}\left(\tau, \pi_{2_{n}}(\tau)\right)-\mathscr{Z}_{1}\left(\tau, \pi_{2}(\tau)\right)\right| \mathrm{d} \tau\right] \mathrm{d} \mathfrak{J} .
\end{aligned}
$$

The continuity of $\mathscr{Z}_{1}$ implies that $\mid \mathscr{Z}_{1}\left(\tau, \pi_{2_{n}}(\tau)-\mathscr{Z}_{1}\left(\tau, \pi_{2}(\tau)\right) \mid \longrightarrow 0\right.$ as $n \longrightarrow \infty$, and then,

$$
\left\|\mathscr{G}_{1}\left(\pi_{2_{n}}\right)(w)-\mathscr{G}_{1}\left(\pi_{2}\right)(w)\right\| \longrightarrow 0, \quad \text { as } n \longrightarrow \infty,
$$

and also, we can in the same way prove that

$$
\left\|\mathscr{G}_{2}\left(\pi_{1_{n}}\right)(w)-\mathscr{G}_{2}\left(\pi_{1}\right)(w)\right\|
$$$$
\leq(q-1) \rho_{2}^{q-2} \int_{0}^{1}\left|G^{\mathrm{Q}_{2}}(w, \mathfrak{J})\right|\left[\frac{1}{\Gamma\left(\theta_{2}\right)} \int_{0}^{\mathfrak{J}}(\mathfrak{I}-\tau)^{\theta_{2}-1} \mid \mathscr{Z}_{2}\left(\tau, \pi_{1_{n}}(\tau)\right)\right.
$$$$
\left.-\mathscr{E}_{2}\left(\tau, \pi_{1}(\tau)\right) \mid \mathrm{d} \tau\right] \mathrm{d} \Im .
$$ 
The continuity of $\mathscr{E}_{2}$ implies that $\mid \mathscr{Z}_{2}\left(\tau, \pi_{1_{n}}(\tau)-\mathscr{E}_{2}\left(\tau, \pi_{1}(\tau)\right) \mid \longrightarrow 0\right.$ as $n \longrightarrow \infty$, and then,

$\left\|\mathscr{G}_{2}\left(\pi_{1_{n}}\right)(w)-\mathscr{G}_{2}\left(\pi_{1}\right)(w)\right\| \longrightarrow 0, \quad$ as $n \longrightarrow \infty$.

Thus, from (49) and (51), we have

$\left\|\mathscr{G}\left(\pi_{1_{n}}, \pi_{2_{n}}\right)(w)-\mathscr{G}\left(\pi_{1}, \pi_{2}\right)(w)\right\|$

$\leq(q-1) \rho_{1}^{q-2} \int_{0}^{1}\left|G^{Q_{1}}(w, \mathfrak{J})\right|\left[\frac{1}{\Gamma\left(\theta_{1}\right)} \int_{0}^{\mathfrak{J}}(\mathfrak{J}-\tau)^{\theta_{1}-1} \mid \mathscr{Z}_{1}\left(\tau, \pi_{2_{n}}(\tau)\right)\right.$

$\left.-\mathscr{Z}_{1}\left(\tau, \pi_{2}(\tau)\right) \mid \mathrm{d} \tau\right] \mathrm{d} \mathfrak{I}$

$+(q-1) \rho_{2}^{q-2} \int_{0}^{1}\left|G^{\mathrm{e}_{2}}(w, \mathfrak{J})\right|\left[\frac{1}{\Gamma\left(\theta_{2}\right)} \int_{0}^{\mathfrak{I}}(\mathfrak{J}-\tau)^{\theta_{2}-1} \mid \mathscr{X}_{2}\left(\tau, \pi_{1_{n}}(\tau)\right)\right.$

$\left.-\mathscr{Z}_{2}\left(\tau, \pi_{1}(\tau)\right) \mid \mathrm{d} \tau\right] \mathrm{d} \Im$.
From the continuity of $\mathscr{Z}_{1}$ and $\mathscr{Z}_{2}$ and (52), we have $\left\|\mathscr{G}\left(\pi_{1_{n}}, \pi_{2_{n}}\right)(w)-\mathscr{G}\left(\pi_{1}, \pi_{2}\right)(w)\right\| \longrightarrow 0 \quad$ as $n \longrightarrow \infty$.

To calculate (46) for $\mathscr{G}$, using assumption $\left(H_{3}\right)$ and (29), we obtain

$$
\begin{aligned}
\left|\mathscr{G}_{1}\left(\pi_{2}\right)(w)\right|= & \left|\int_{0}^{1} \mathscr{G}^{\varrho_{1}}(w, \mathfrak{I}) \phi_{q}\left(\frac{1}{\Gamma\left(\theta_{1}\right)} \int_{0}^{\mathfrak{I}}(\mathfrak{I}-\tau)^{\theta_{1}-1} \mathscr{Z}_{1}\left(\tau, \pi_{2}(\tau) \mathrm{d} \tau\right) \mathrm{d} \mathfrak{I}\right)\right| \\
& \leq \int_{0}^{1}\left|G^{\varrho_{1}}(w, \mathfrak{I})\right| \phi_{q}\left(\frac{1}{\Gamma\left(\theta_{1}\right)} \int_{0}^{\mathfrak{I}}(\mathfrak{I}-\tau)^{\theta_{1}-1}\left|\mathscr{Z}_{1}\left(\tau, \pi_{2}(\tau)\right)\right| \mathrm{d} \tau\right) \mathrm{d} \mathfrak{I} \\
& \leq \int_{0}^{1}\left|G^{\varrho_{1}}(w, \mathfrak{I})\right| \phi_{q}\left(\frac{1}{\Gamma\left(\theta_{1}\right)} \int_{0}^{\mathfrak{I}}(\mathfrak{I}-\tau)^{\theta_{1}-1} \phi_{p}\left(g\left|\pi_{2}\right|^{q_{2}}+N_{\mathscr{I}_{1}}\right)\right) \mathrm{d} \mathfrak{I} \\
& \leq\left(\frac{1}{\Gamma\left(\varrho_{1}+1\right)}+\frac{\Gamma\left(\varrho_{1}-\lambda-2\right)}{\Gamma\left(\varrho_{1}-2\right) \Gamma\left(\varrho_{1}-\lambda+1\right)}\right)\left[\frac{1}{\Gamma\left(\theta_{1}+1\right)}\right]^{q-1}\left(g\left|\pi_{2}\right|^{q_{2}}+N_{\mathscr{Z}_{1}}\right) .
\end{aligned}
$$

From assumption $\left(\mathrm{H}_{3}\right)$ and (29), we get

$$
\begin{aligned}
\left|\mathscr{G}_{2}\left(\pi_{1}\right)(w)\right|= & \left|\int_{0}^{1} \mathscr{G}^{\varrho_{2}}(w, \mathfrak{I}) \phi_{q}\left(\frac{1}{\Gamma\left(\theta_{2}\right)} \int_{0}^{\mathfrak{I}}(\mathfrak{I}-\tau)^{\theta_{2}-1} \mathscr{Z}_{2}\left(\tau, \pi_{1}(\tau) d \tau\right)\right) \mathrm{d} \mathfrak{J}\right| \\
& \leq \int_{0}^{1}\left|G^{\varrho_{2}}(w, \mathfrak{J})\right| \phi_{q}\left(\frac{1}{\Gamma\left(\theta_{2}\right)} \int_{0}^{\mathfrak{I}}(\mathfrak{I}-\tau)^{\theta_{2}-1}\left|\mathscr{Z}_{2}\left(\tau, \pi_{1}(\tau)\right)\right| \mathrm{d} \tau\right) \mathrm{d} \mathfrak{I} \\
& \leq \int_{0}^{1}\left|G^{\varrho_{2}}(w, \mathfrak{I})\right| \phi_{q}\left(\frac{1}{\Gamma\left(\theta_{2}\right)} \int_{0}^{\mathfrak{I}}(\mathfrak{I}-\tau)^{\theta_{2}-1} \phi_{p}\left(h\left|\pi_{1}\right|^{q_{2}}+N_{\mathscr{I}_{2}}\right) \mathrm{d} \tau\right) \mathrm{d} \mathfrak{I} .
\end{aligned}
$$

Then,

$$
\begin{aligned}
\left|\mathscr{G}_{2}\left(\pi_{1}\right)(w)\right| & \leq \int_{0}^{1}\left|G^{\varrho_{2}}(w, \mathfrak{J})\right| \phi_{q}\left(\frac{1}{\Gamma\left(\theta_{2}\right)} \int_{0}^{\mathfrak{J}}(\mathfrak{I}-\tau)^{\theta_{2}-1} \phi_{p}\left(h\left|\pi_{1}\right|^{q_{2}}+N_{\mathscr{Z}_{2}}\right) \mathrm{d} \tau\right) \mathrm{d} \mathfrak{I} \\
& \leq\left(\frac{1}{\Gamma\left(\varrho_{2}+1\right)}+\frac{\Gamma\left(\varrho_{2}-\sigma-2\right)}{\Gamma\left(\varrho_{2}-2\right) \Gamma\left(\varrho_{2}-\sigma+1\right)}\right)\left[\frac{1}{\Gamma\left(\theta_{2}+1\right)}\right]^{q-1}\left(h\left|\pi_{1}\right|^{q_{2}}+N_{\mathscr{Z}_{2}}\right) .
\end{aligned}
$$


By the help of (54) and (55), we have obtained

$$
\begin{aligned}
\left\|\mathscr{G}\left(\pi_{1}, \pi_{2}\right)\right\| & =\left\|\mathscr{G}_{1}\left(\pi_{2}\right)\right\|+\left\|\mathscr{G}_{2}\left(\pi_{1}\right)\right\| \\
& \leq \gamma\left(g\left\|\pi_{2}\right\|^{q_{2}}+N_{\mathscr{I}_{1}}\right)+\gamma\left(h\left\|\pi_{1}\right\|^{q_{2}}+N_{\mathscr{I}_{2}}\right) \\
& \leq \gamma(g+h)\left(\left\|\pi_{2}\right\|^{q_{2}}+\left\|\pi_{1}\right\|^{q_{2}}\right)+\gamma\left(N_{\mathscr{Z}_{1}}+N_{\mathscr{Z}_{2}}\right) \\
& =\Lambda\left\|\left(\pi_{1}, \pi_{2}\right)\right\|^{q_{2}}+\Theta .
\end{aligned}
$$

Theorem 5. The operator $\mathscr{G}: \Omega \longrightarrow \Omega$ is 9 -Lipschitz with constant zero and is compact.

Proof. Take a bounded set $E$ and a sequence $\left(\pi_{1_{n}}, \pi_{2_{n}}\right)$ such that $E \subset \mathfrak{B}_{r} \subseteq \Omega$. Then, using (46), we have

$$
\left\|\mathscr{G}\left(\pi_{1_{n}}, \pi_{2_{n}}\right)\right\| \leq \Lambda\left\|\left(\pi_{1}, \pi_{2}\right)\right\|+\Theta, \quad \text { for all }\left(\pi_{1}, \pi_{2}\right) \in \Omega,
$$

which means that $\mathscr{G}$ is bounded. Now, for all $\left(\pi_{1_{n}}, \pi_{2_{n}}\right) \in E$, we have, for $0 \leq w_{1}<w_{2} \leq 1$,

$$
\begin{aligned}
& \left|\mathscr{G}_{1} \pi_{2_{n}}\left(w_{1}\right)-\mathscr{G}_{1} \pi_{2_{n}}\left(w_{2}\right)\right| \\
& =\mid \int_{0}^{1} G^{Q_{1}}\left(w_{1}, \mathfrak{I}\right) \phi_{q}\left(\frac{1}{\Gamma\left(\theta_{1}\right)} \int_{0}^{\mathfrak{I}}(\mathfrak{I}-\tau)^{\theta_{1}-1} \mathscr{Z}_{1}\left(\left(\tau, \pi_{2}(\tau)\right) \mathrm{d} \tau\right) \mathrm{d} \mathfrak{I}\right) \\
& -\int_{0}^{1} G^{\varrho_{1}}\left(w_{2}, \mathfrak{J}\right) \phi_{q}\left(\frac{1}{\Gamma\left(\theta_{1}\right)} \int_{0}^{\mathfrak{I}}(\mathfrak{I}-\tau)^{\theta_{1}-1} \mathscr{Z}_{1}\left(\left(\tau, \pi_{2}(\tau)\right) \mathrm{d} \tau\right) \mathrm{d} \mathfrak{I}\right) \mid \\
& \leq \int_{0}^{1}\left|G^{\mathrm{Q}_{1}}\left(w_{1}, \mathfrak{J}\right)-G^{\mathrm{Q}_{1}}\left(w_{2}, \mathfrak{J}\right)\right| \phi_{q}\left(\frac{1}{\Gamma\left(\theta_{1}\right)} \int_{0}^{\mathfrak{I}}(\mathfrak{I}-\tau)^{\theta_{1}-1} \phi_{p}\left(g\left\|\pi_{1}\right\|^{q_{2}}+N_{\mathscr{X}_{1}}\right) \mathrm{d} \tau\right) \mathrm{d} \mathfrak{I} .
\end{aligned}
$$

Hence, it follows that

$$
\begin{aligned}
\left|\mathscr{G}_{1} \pi_{2_{n}}\left(w_{1}\right)-\mathscr{G}_{1} \pi_{2_{n}}\left(w_{2}\right)\right| \\
\leq\left[\frac{\left(w_{1}^{\varrho_{1}}-w_{2}^{\varrho_{1}}\right)}{\Gamma\left(\varrho_{1}+1\right)}+\frac{\left(w_{1}^{\varrho_{1}-3}-w_{2}^{\varrho_{1}-3}\right)}{\Gamma\left(\varrho_{1}-2\right) \Gamma\left(\varrho_{1}-\lambda+1\right)}\right]\left[\frac{1}{\Gamma\left(\theta_{1}+1\right)}\right]^{q-1} \\
\quad \times\left(g\left\|\pi_{1}\right\|^{q_{2}}+N_{\mathscr{Z}_{1}}\right) .
\end{aligned}
$$

Similarly, we have

$$
\begin{aligned}
& \left|\mathscr{G}_{2} \pi_{1_{n}}\left(w_{1}\right)-\mathscr{G}_{2} \pi_{1_{n}}\left(w_{2}\right)\right| \\
& \leq\left[\frac{\left(w_{1}^{\varrho_{2}}-w_{2}^{\varrho_{2}}\right)}{\Gamma\left(\varrho_{2}+1\right)}+\frac{\left(w_{1}^{\varrho_{2}-3}-w_{2}^{\varrho_{2}-3}\right)}{\Gamma\left(\varrho_{2}-2\right) \Gamma\left(\varrho_{2}-\sigma+1\right)}\right]\left[\frac{1}{\Gamma\left(\theta_{2}+1\right)}\right]^{q-1} \\
& \quad \times\left(h\left\|\pi_{2}\right\|^{q_{2}}+N_{\mathscr{E}_{2}}\right) .
\end{aligned}
$$

Both the right sides of (55) and (61) tend to be zero as $w_{1} \longrightarrow w_{2}$. Therefore, the operators $\mathscr{G}_{1}$ and $\mathscr{G}_{2}$ are equicontinuous, and hence, $\mathscr{G}=\left(\mathscr{G}_{1}, \mathscr{G}_{2}\right)$ is equicontinuous on $E$. Thus, $\mathscr{G}(E)$ is compact by the theorem of Arzela-Ascoli. Moreover, through Proposition 3, $\mathscr{G}$ is 9 -Lipschitz with constant zero.

Theorem 6. Suppose that $\left(H_{1}\right)-\left(H_{3}\right)$ are satisfied with $\Lambda+C_{\mathscr{F}} \leq 1$. Then, the toppled system (5) has at least one solution $\left(\pi_{1}, \pi_{2}\right) \in \Omega$. Furthermore, the set of solutions of (5) is bounded in $\Omega$.
Proof. With the help of Theorem 3, F is 9 -Lipschitz with constant $0 \leq C_{\mathscr{F}}<1$, and $\mathscr{G}$ is $\vartheta$-Lipschitz with constant zero by Theorem 5 . Thus, $\mathscr{T}$ is strictly 9 -condensing with constant $\eta$ by Proposition 2. Now, let us set that we have to show that $\mathfrak{B}$ is bounded in $\Omega$. In fact,

$$
\begin{aligned}
\left\|\left(\pi_{1}, \pi_{2}\right)\right\|= & \left\|\kappa \mathscr{T}\left(\pi_{1}, \pi_{2}\right)\right\| \leq\left\|\mathscr{T}\left(\pi_{1}, \pi_{2}\right)\right\| \\
\leq & \left(\left\|\mathscr{F}\left(\pi_{1}, \pi_{2}\right)\right\|+\left\|\mathscr{G}\left(\pi_{1}, \pi_{2}\right)\right\|\right) \\
\leq & C_{\mathscr{F}}\left\|\left(\pi_{1}, \pi_{2}\right)\right\|^{q_{1}}+N_{\mathscr{F}}+\Lambda\left\|\left(\pi_{1}, \pi_{2}\right)\right\|^{q_{2}}+\Theta \\
= & \left(C_{\mathscr{F}}+\Lambda\right)\left\|\left(\pi_{1}, \pi_{2}\right)\right\|^{q_{3}}+N_{\mathscr{F}}+\Theta, \\
& \text { where } q_{3}=\max \left\{q_{1}, q_{2}\right\} .
\end{aligned}
$$

Obviously, $\left\|\pi_{1}, \pi_{2}\right\|$ is bounded. If not correct, take $\left\|\pi_{1}, \pi_{2}\right\|=\mathcal{S}$ such that $\mathcal{S} \longrightarrow \infty$ and $q_{3} \in(0,1)$. Consequently,

$$
\begin{aligned}
& 1 \leq\left(C_{\mathscr{F}}+\Lambda\right) \frac{\left\|\left(\pi_{1}, \pi_{2}\right)\right\|^{q_{3}}}{\left\|\left(\pi_{1}, \pi_{2}\right)\right\|}+\frac{N_{\mathscr{F}}+\Theta}{\left\|\left(\pi_{1}, \pi_{2}\right)\right\|}, \\
& 1 \leq \frac{\left(C_{\mathscr{F}}+\Lambda\right) \mathcal{S}^{q_{3}}}{\mathcal{S}}+\frac{N_{\mathscr{F}}+\Theta}{\mathcal{S}}, \\
& 1 \leq \frac{\left(C_{\mathscr{F}}+\Lambda\right)}{\mathcal{S}^{1-q_{3}}}+\frac{N_{\mathscr{F}}+\Theta}{\mathcal{S}} \longrightarrow 0 \text { as } \mathcal{S} \longrightarrow \infty,
\end{aligned}
$$

which is a contradiction. So, $\mathfrak{B}$ is bounded. Thus, by Theorem 7 , we conclude that $\mathscr{T}$ has at least one fixed point and that is a solution of system (5), and the set of solutions is bounded in $\Omega$. 
Theorem 7. Suppose that $\left(H_{1}\right)-\left(H_{4}\right)$ hold and $\chi<1$, where

$$
\begin{aligned}
\chi= & \frac{K_{\varphi} \Gamma\left(\varrho_{1}-\lambda-2\right) W^{\lambda}}{\Gamma\left(\varrho_{1}-2\right) \Gamma(\lambda+1)}+\frac{K_{\omega} \Gamma\left(\varrho_{2}-\sigma-2\right) W^{\sigma}}{\Gamma\left(\varrho_{2}-2\right) \Gamma(\sigma+1)} \\
& +(q-1) \rho_{1}^{q-2} L_{\mathscr{Z}_{1}}\left(\frac{1}{\Gamma\left(\varrho_{1}+1\right)}+\frac{\Gamma\left(\varrho_{1}-\lambda-2\right)}{\Gamma\left(\varrho_{1}-2\right) \Gamma\left(\varrho_{1}-\lambda+1\right)}\right)\left(\frac{1}{\Gamma\left(\theta_{1}+1\right)}\right) \\
& +(q-1) \rho_{2}^{q-2} L_{\mathscr{Z}_{2}}\left(\frac{1}{\Gamma\left(\varrho_{2}+1\right)}+\frac{\Gamma\left(\varrho_{2}-\sigma-2\right)}{\Gamma\left(\varrho_{2}-2\right) \Gamma\left(\varrho_{2}-\sigma+1\right)}\right)\left(\frac{1}{\Gamma\left(\theta_{2}+1\right)}\right) .
\end{aligned}
$$

Then, toppled system (5) has a unique solution.

$$
\begin{aligned}
\left|\mathscr{T}\left(\pi_{1}, \pi_{2}\right)-\mathscr{T}\left(\overline{\pi_{1}}, \overline{\pi_{2}}\right)\right| & =\left|\left[\mathscr{F}\left(\pi_{1}, \pi_{2}\right)+\mathscr{G}\left(\pi_{1}, \pi_{2}\right)\right]-\left[\mathscr{F}\left(\overline{\pi_{1}}, \overline{\pi_{2}}\right)+\mathscr{G}\left(\overline{\pi_{1}}, \overline{\pi_{2}}\right)\right]\right| \\
& \leq\left|\mathscr{F}\left(\pi_{1}, \pi_{2}\right)-\mathscr{F}\left(\overline{\pi_{1}}, \overline{\pi_{2}}\right)\right|+\left|\mathscr{G}\left(\pi_{1}, \pi_{2}\right)-\mathscr{G}\left(\overline{\pi_{1}}, \overline{\pi_{2}}\right)\right|,
\end{aligned}
$$

and after simplification, we obtain

$$
\begin{aligned}
&\left\|\mathscr{T}\left(\pi_{1}, \pi_{2}\right)-\mathscr{T}\left(\overline{\pi_{1}}, \overline{\pi_{2}}\right)\right\| \\
& \leq\left(\frac{K_{\varphi} \Gamma\left(\varrho_{1}-\lambda-2\right) W^{\lambda}}{\Gamma\left(\varrho_{1}-2\right) \Gamma(\lambda+1)}+\frac{K_{\omega} \Gamma\left(\varrho_{2}-\sigma-2\right) W^{\sigma}}{\Gamma\left(\varrho_{2}-2\right) \Gamma(\sigma+1)}\right. \\
&+(q-1) \rho_{1}^{q-2} L_{\mathscr{I}_{1}}\left(\frac{1}{\Gamma\left(\varrho_{1}+1\right)}+\frac{\Gamma\left(\varrho_{1}-\lambda-2\right)}{\Gamma\left(\varrho_{1}-2\right) \Gamma\left(\varrho_{1}-\lambda+1\right)}\right)\left(\frac{1}{\Gamma\left(\theta_{1}+1\right)}\right) \\
&\left.+(q-1) \rho_{2}^{q-2} L_{\mathscr{I}_{2}}\left(\frac{1}{\Gamma\left(\varrho_{2}+1\right)}+\frac{\Gamma\left(\varrho_{2}-\sigma-2\right)}{\Gamma\left(\varrho_{2}-2\right) \Gamma\left(\varrho_{2}-\sigma+1\right)}\right)\left(\frac{1}{\Gamma\left(\theta_{2}+1\right)}\right)\right) \\
& \quad \times\left\|\left(\pi_{1}, \pi_{2}\right)-\left(\overline{\pi_{1}}, \overline{\pi_{2}}\right)\right\|,
\end{aligned}
$$

which implies that

$$
\left\|\mathscr{T}\left(\pi_{1}, \pi_{2}\right)-\mathscr{T}\left(\overline{\pi_{1}}, \overline{\pi_{2}}\right)\right\| \leq \chi\left\|\left(\pi_{1}, \pi_{2}\right)-\left(\overline{\pi_{1}}, \overline{\pi_{2}}\right)\right\| .
$$

Thus, the operator $\mathscr{T}$ is a contraction as $\chi<1$, and by the Banach fixed-point theorem, $\mathscr{T}$ has a unique fixed point, and then, considered toppled system (5) has a unique solution.

\section{Hyers-Ulam Stability}

In this section, we investigate the stability of Hyers-Ulam for the suggested toppled system.

Definition 7. We say that the toppled system of Hammerstein-kind integral equations (27) is Hyers-Ulam stable if 
there exists positive constants $a, b, c$, and $d$ such that, for each $\xi_{1}, \xi_{2}>0$ and any solution $\left(\pi_{1}^{*}, \pi_{2}^{*}\right)$ of the system

$$
\left\{\begin{array}{l}
\mid \pi_{1}^{*}(w)-\frac{\Gamma\left(\varrho_{1}-\lambda-2\right) w^{\varrho_{1}-3}}{\Gamma\left(\varrho_{1}-2\right) \Gamma(\lambda)} \int_{0}^{W}(W-\mathfrak{I})^{\lambda-1} \varphi\left(\pi_{1}^{*}(\mathfrak{I})\right) d \mathfrak{I} \\
\quad+\int_{0}^{1} G^{\varrho_{1}}(w, \mathfrak{J}) \phi_{q}\left(\frac{1}{\Gamma\left(\theta_{1}\right)} \int_{0}^{\mathfrak{J}}(\mathfrak{I}-\tau)^{\theta_{1}-1} \mathscr{Z}_{1}\left(\tau, \pi_{2}^{*}(\tau)\right) d \tau\right) d \mathfrak{J} \mid \leq \xi_{1}, \\
\mid \pi_{2}^{*}(w)-\frac{\Gamma\left(\varrho_{2}-\sigma-2\right) w^{\varrho_{2}-3}}{\Gamma\left(\varrho_{2}-2\right) \Gamma(\sigma)} \int_{0}^{W}(W-\mathfrak{J})^{\sigma-1} \Phi\left(\pi_{2}^{*}(\mathfrak{I})\right) d \mathfrak{I} \\
\quad+\int_{0}^{1} G^{\varrho_{2}}(w, \mathfrak{J}) \phi_{q}\left(\frac{1}{\Gamma\left(\theta_{2}\right)} \int_{0}^{\mathfrak{J}}(\mathfrak{I}-\tau)^{\theta_{2}-1} \mathscr{Z}_{2}\left(\tau, \pi_{1}^{*}(\tau)\right) d \tau\right) d \mathfrak{I} \mid \leq \xi_{2},
\end{array}\right.
$$

there exists $\left(\pi_{1}, \pi_{2}\right)$ which is the unique solution of (27) satisfying that

$$
\begin{aligned}
& \left|\pi_{1}(w)-\pi_{1}^{*}(w)\right| \leq a \xi_{1}+b \xi_{2} \\
& \left|\pi_{2}(w)-\pi_{2}^{*}(w)\right| \leq c \xi_{1}+d \xi_{2}
\end{aligned}
$$

Theorem 8. The toppled system (5) is Hyers-Ulam stable under hypotheses $\left(H_{1}\right)-\left(H_{4}\right)$.

Proof. With the help of Definition 7 and Theorem 7, suppose that $\left(\pi_{1}, \pi_{2}\right)$ to be the correct solution and the pair $\left(\pi_{1}^{*}, \pi_{2}^{*}\right)$ be the other solution of system (27). Then, we have, from the first equation of (27),

$$
\begin{aligned}
& \left|\pi_{1}(w)-\pi_{1}^{*}(w)\right|=\mid \frac{\Gamma\left(\varrho_{1}-\lambda-2\right) w^{\varrho_{1}-3}}{\Gamma\left(\varrho_{1}-2\right) \Gamma(\lambda)} \int_{0}^{W}(W-\mathfrak{J})^{\lambda-1} \varphi\left(\pi_{1}(\mathfrak{I}) \mathrm{d} \mathfrak{I}\right. \\
& +\int_{0}^{1} G^{Q_{1}}(w, \mathfrak{J}) \phi_{q}\left(\frac{1}{\Gamma\left(\theta_{1}\right)} \int_{0}^{\mathfrak{J}}(\mathfrak{\Im}-\tau)^{\theta_{1}-1} \mathscr{Z}_{1}\left(\tau, \pi_{2}(\tau)\right) \mathrm{d} \tau\right) \mathrm{d} \mathfrak{\Im} \\
& -\frac{\Gamma\left(\varrho_{1}-\lambda-2\right)}{\Gamma\left(\varrho_{1}-2\right) \Gamma(\lambda)} \int_{0}^{W}(W-\mathfrak{J})^{\lambda-1} \varphi\left(\pi_{1}^{*}(\mathfrak{I}) \mathrm{d} \mathfrak{I}\right. \\
& -\int_{0}^{1} G^{\varrho_{1}}(w, \mathfrak{I}) \phi_{q}\left(\frac{1}{\Gamma\left(\theta_{1}\right)} \int_{0}^{\mathfrak{J}}(\mathfrak{I}-\tau)^{\theta_{1}-1} \mathscr{Z}_{1}\left(\tau, \pi_{2}^{*}(\tau)\right) \mathrm{d} \tau\right) \mathrm{d} \mathfrak{I} \mid \\
& \leq \frac{\Gamma\left(\varrho_{1}-\lambda-2\right)}{\Gamma\left(\varrho_{1}-2\right) \Gamma(\lambda)} \int_{0}^{W}(W-\mathfrak{J})^{\lambda-1}\left(\varphi\left(\pi_{1}\right)-\varphi\left(\pi_{1}^{*}\right)\right) \mathrm{d} \mathfrak{I} \\
& +\int_{0}^{1}\left\|G^{\varrho_{1}}(w, \mathfrak{J})\right\| \phi_{q}\left[\frac{1}{\Gamma\left(\theta_{1}\right)} \int_{0}^{\mathfrak{I}}(\mathfrak{I}-\tau)^{\theta_{1}-1} \mathscr{Z}_{1}\left(\tau, \pi_{2}(\tau)\right) \mathrm{d} \tau\right] \\
& -\phi_{q}\left[\frac{1}{\Gamma\left(\theta_{1}\right)} \int_{0}^{\mathfrak{I}}(\mathfrak{\Im}-\tau)^{\theta_{1}-1} \mathscr{Z}_{1}\left(\tau, \pi_{2}^{*}(\tau)\right) \mathrm{d} \tau\right] \mid \mathrm{d} \mathfrak{I} \\
& \leq \frac{\Gamma\left(\varrho_{1}-\lambda-2\right)}{\Gamma\left(\varrho_{1}-2\right) \Gamma(\lambda)} \int_{0}^{W}(W-\mathfrak{J})^{\lambda-1}\left|\left(\varphi\left(\pi_{1}\right)-\varphi\left(\pi_{1}^{*}\right)\right)\right| \mathrm{d} \mathfrak{\Im} \\
& +(q-1) \rho_{1}^{q-2} \int_{0}^{1}\left|G^{Q_{1}}(w, \mathfrak{J})\right|\left(\frac{1}{\Gamma\left(\theta_{1}\right)} \int_{0}^{\mathfrak{I}}(\mathfrak{I}-\tau)^{\theta_{1}-1}\right. \\
& \left.\times\left|\mathscr{Z}_{1}\left(\tau, \pi_{2}(\tau)\right)-\mathscr{Z}_{1}\left(\tau, \pi_{2}^{*}(\tau)\right)\right| \mathrm{d} \tau\right) \mathrm{d} \Im .
\end{aligned}
$$


Then,

$$
\begin{aligned}
\left|\pi_{1}(w)-\pi_{1}^{*}(w)\right| \leq & \frac{\Gamma\left(\varrho_{1}-\lambda-2\right) W^{\lambda} K_{\varphi}}{\Gamma\left(\varrho_{1}-2\right) \Gamma(\lambda+1)}\left\|\pi_{1}(w)-\pi_{1}^{*}(w)\right\| \\
& +(q-1) \rho_{1}^{q-2} L_{\mathscr{Z}_{1}}\left(\frac{1}{\Gamma\left(\varrho_{1}+1\right)}+\frac{\Gamma\left(\varrho_{1}-\lambda-2\right)}{\Gamma\left(\varrho_{1}-2\right) \Gamma\left(\varrho_{1}-\lambda+1\right)}\right)\left(\frac{1}{\Gamma\left(\theta_{1}+1\right)}\right) \\
& \times\left\|\pi_{2}(w)-\pi_{2}^{*}(w)\right\| \leq a \xi_{1}+b \xi_{2},
\end{aligned}
$$

where

$$
\begin{aligned}
& a=\frac{\Gamma\left(\varrho_{1}-\lambda-2\right) W^{\lambda} K_{\varphi}}{\Gamma\left(\varrho_{1}-2\right) \Gamma(\lambda+1)} \\
& b=(q-1) \rho_{1}^{q-2} L_{\mathscr{L}_{1}}\left(\frac{1}{\Gamma\left(\varrho_{1}+1\right)}+\frac{\Gamma\left(\varrho_{1}-\lambda-2\right)}{\Gamma\left(\varrho_{1}-2\right) \Gamma\left(\varrho_{1}-\lambda+1\right)}\right)\left(\frac{1}{\Gamma\left(\theta_{1}+1\right)}\right) .
\end{aligned}
$$

Similarly, we get

$$
\left|\pi_{2}(w)-\pi_{2}^{*}(w)\right| \leq c \xi_{1}+d \xi_{2},
$$

where

$$
\begin{aligned}
& c=\frac{\Gamma\left(\varrho_{2}-\sigma-2\right) W^{\sigma} K_{\varpi}}{\Gamma\left(\varrho_{2}-2\right) \Gamma(\sigma+1)} \\
& d=(q-1) \rho_{2}^{q-2} L_{\mathscr{L}_{2}}\left(\frac{1}{\Gamma\left(\varrho_{2}+1\right)}+\frac{\Gamma\left(\varrho_{2}-\sigma-2\right)}{\Gamma\left(\varrho_{2}-2\right) \Gamma\left(\varrho_{2}-\sigma+1\right)}\right)\left(\frac{1}{\Gamma\left(\theta_{2}+1\right)}\right) .
\end{aligned}
$$

Hence, by (71) and (73), integral equations' toppled system (27) is Hyers-Ulam stable. Thus, proposed toppled system (5) is Hyers-Ulam stable.

\section{Illustrative Example}

In this section, we introduce an application of our results, which were proved in Sections 3 and 4.

Example 1. Consider the following toppled fractional system with the $p$-Laplacian operator and integral boundary conditions for $n=5$ :

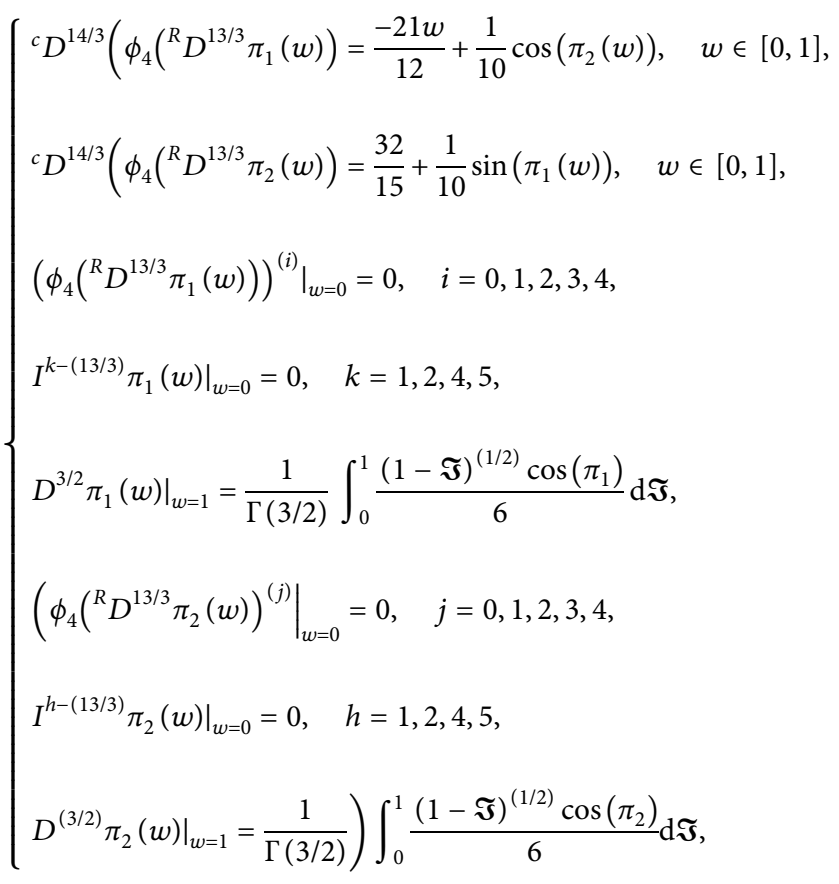


where $\theta_{1}=\theta_{2}=(14 / 3), p=4, \varrho_{1}=\varrho_{2}=(13 / 3)$, and $\lambda=$ $\sigma=(3 / 2)$. Then, we obtain $K_{\varphi}=K_{\oplus}=(1 / 6)$ and $L_{\mathscr{E}_{1}}=L_{\mathscr{Z}_{2}}=(1 / 10)$. Via simple calculation and taking $\rho_{1}=\rho_{2}=(1 / 2)$, we get $\chi=0.1767<1$. Hence, by Theorem 7 , toppled system (75) has a unique solution. With comparable fashion, it is easy to verify the fulfillment of the conditions of Theorem 6. Likewise, the conditions of Theorem 8 can be easily confirmed, and consequently, the solution of system (75) is Hyers-Ulam stable.

\section{Conclusion}

In this study, we analyzed the stability and uniqueness solution of Caputo and Riemann-Liouville fractional derivatives with fractional orders $n-1<\theta_{1}, \theta_{2}, \varrho_{1}, \varrho_{2} \leq n$, and $n \geq 3$. By using the topological degree theory, we have proved sufficient conditions for the EUS of the coupled system of fractional differential equations with integral boundary conditions involving the $p$-Laplacian operator. Also, we have found appropriate conditions for Hyers-Ulam stability of the solution for the considered system. At the end, we have provided an example that supported our results as we have done in Section 5 to confirm the theoretical analysis.

\section{Data Availability}

No data were used to support this study.

\section{Conflicts of Interest}

The authors declare that they have no conflicts of interest.

\section{Acknowledgments}

This work was supported by the National Natural Science Foundation of China (no. 11871475).

\section{References}

[1] R. P. Agarwal, D. O’Regan, and S. Staněk, "Positive solutions for mixed problems of singular fractional differential equations," Mathematische Nachrichten, vol. 285, no. 1, pp. 27-41, 2012.

[2] W. Al-Sadi, H. Zhenyou, and A. Alkhazzan, "Existence and stability of a positive solution for nonlinear hybrid fractional differential equations with singularity," Journal of Taibah University for Science, vol. 13, no. 1, pp. 951-960, 2019.

[3] A. Hallaci, H. Boulares, and M. Kurulay, "On the study of nonlinear fractional differential equations on unbounded interval," General Letters in Mathematics, vol. 5, no. 3, pp. 111-117, 2018.

[4] H. Jafari, H. Jassim, S. P. Moshokoa, V. M. Ariyan, and F. Tchier, "Reduced differential transform method for partial differential equations within local fractional derivative operators," Advances in Mechanical Engineering, vol. 8, no. 4, Article ID 1687814016633013, 2016.

[5] A. A. Kilbas, H. M. Srivastava, and J. J. Trujillo, "Theory and applications of fractional differential equations," North-Holland. Mathematics Studies, vol. 204, 2006.

[6] C. Kou, H. Zhou, and Y. Yan, "Existence of solutions of initial value problems for nonlinear fractional differential equations on the half-axis," Nonlinear Analysis: Theory, Methods and Applications, vol. 74, no. 17, pp. 5975-5986, 2011.

[7] C. P. Li and F. R. Zhang, "A survey on the stability of fractional differential equations," The European Physical Journal Special Topics, vol. 193, no. 1, pp. 27-47, 2011.

[8] I. Podlubny, Fractional Differential Equations, Mathematics in Science and Engineering, vol. 198, , 1999.

[9] B. Zhou, L. Zhang, E. Addai, and N. Zhang, "Multiple positive solutions for nonlinear high-order riemann-liouville fractional differential equations boundary value problems with p-laplacian operator," Boundary Value Problems, vol. 2020, no. 1, p. 26, 2020.

[10] M. A. Dokuyucu, E. Celik, H. Bulut, and H. M. Baskonus, "Cancer treatment model with the Caputo-Fabrizio fractional derivative," The European Physical Journal Plus, vol. 133, no. 3, pp. 1-6, 2018.

[11] M. A. Dokuyucu, "A fractional order alcoholism model via Caputo-Fabrizio derivative," AIMS Mathematics, vol. 5, no. 2, pp. 781-797, 2020.

[12] K. İlknur, E. Akçetin, and P. Yaprakdal, "Numerical approximation for the spread of SIQR model with Caputo fractional order derivative," Turkish Journal of Science, vol. 5, no. 2, pp. 124-139, 2020.

[13] M. Goyal, H. M. Baskonus, and A. Prakash, "Regarding new positive, bounded and convergent numerical solution of nonlinear time fractional HIV/AIDS transmission model," Chaos, Solitons and Fractals, vol. 139, Article ID 110096, 2020.

[14] M. A. Dokuyucu, "Caputo and atangana-baleanu-caputo fractional derivative applied to garden equation"” Turkish Journal of Science, vol. 5, no. 1, pp. 1-7, 2020.

[15] B. Li and H. Gou, "Existence of solutions for impulsive fractional evolution equations with periodic boundary condition," Advances in Difference Equations, vol. 2017, no. 1, pp. 1-22, 2017.

[16] M. M. Matar, I. A. Amra, and J. Alzabut, "Existence of solutions for tripled system of fractional differential equations involving cyclic permutation boundary conditions," Boundary Value Problems, vol. 2020, no. 1, pp. 1-13, 2020.

[17] J. A. Nanware and D. B. Dhaigude, "Existence and uniqueness of solutions of differential equations of fractional order with integral boundary conditions," Journal of Nonlinear Sciences and Applications, vol. 7, no. 4, pp. 246-254, 2014.

[18] H. Yang, R. P. Agarwal, and H. K. Nashine, "Coupled fixed point theorems with applications to fractional evolution equations," Advances in Difference Equations, vol. 2017, no. 1, p. 239, 2017.

[19] Z. Ali, A. Zada, and K. Shah, "On ulam's stability for a coupled systems of nonlinear implicit fractional differential equations," Bulletin of the Malaysian Mathematical Sciences Society, vol. 42, no. 5, pp. 2681-2699, 2019.

[20] K. Shah, "Nonlocal boundary value problems for nonlinear toppled system of fractional differential equations," Hacettepe Journal of Mathematics and Statistics, vol. 49, no. 1, pp. 316-337, 2020.

[21] K. Shah, A. Ali, and R. A. Khan, "Degree theory and existence of positive solutions to coupled systems of multi-point boundary value problems," Boundary Value Problems, vol. 2016, no. 1, pp. 1-12, 2016.

[22] Y. Tian, Y. Wei, and S. Sun, "Multiplicity for fractional differential equations with p-laplacian," Boundary Value Problems, vol. 2018, no. 1, p. 127, 2018.

[23] J. R. L. Webb, "Positive solutions of some higher order nonlocal boundary value problems," Electronic Journal of 
Qualitative Theory of Differential Equations, vol. 29, pp. 1-15, 2010.

[24] H. Khan, W. Chen, A. Khan, T. S. Khan, and Q. M. Al-Madlal, "Hyers-ulam stability and existence criteria for coupled fractional differential equations involving p-laplacian operator," Advnces in Difference Equations, vol. 1, p. 455, 2018.

[25] A. Khan, K. Shah, Y. Liand, and T. S. Khan, "Ulam type stability for a coupled system of boundary value problems of nonlinear fractional differential equations," Journal of Function Spaces, vol. 2017, Article ID 3046013, 8 pages, 2017.

[26] R. A. Khan and K. Shah, "Multiple positive solutions to a coupled systems of nonlinear fractional differential equations," SpringerPlus, vol. 5, no. 1, pp. 1-20, 2016.

[27] K. Shah and R. A. Khan, "Existence and uniqueness of positive solutions to a coupled system of nonlinear fractional order differential equations with anti periodic boundary conditions," Differential Equations and Applications, vol. 7, no. 2, pp. 245-262, 2015.

[28] K. Shah, H. Khalil, and R. A. Khan, "Investigation of positive solution to a coupled system of impulsive boundary value problems for nonlinear fractional order differential equations," Chaos, Solitons and Fractals, vol. 77, pp. 240-246, 2015.

[29] F. Isaia, "On a nonlinear integral equation without compactness," Acta Mathematica Universitatis Comenianae, vol. 75, no. 2, pp. 233-240, 2006.

[30] J. Wang, Y. Zhou, and W. Wei, "Study in fractional differential equations by means of topological degree methods," $\mathrm{Nu}$ merical Functional Analysis and Optimization, vol. 33, no. 2, pp. 216-238, 2012.

[31] K. Shah and R. A. Khan, "Existence and uniqueness results to a coupled system of fractional order boundary value problems by topological degree theory," Numerical Functional Analysis and Optimization, vol. 37, no. 7, pp. 887-899, 2016.

[32] H. Khan, Y. Li, H. Sun, and A. Khan, "Existence of solution and Hyers-Ulam stability for a coupled system of fractional differential equations with p-Laplacian operator," Journal of Nonlinear Sciences and Applications, vol. 10, no. 10, pp. 5219-5229, 2017.

[33] N. Ahmad, Z. Ali, K. Khan, A. Zada, and R. Ghaus, "Analysis of implicit type nonlinear dynamical problem of impulsive fractional differential equations," Complexity, vol. 2018, Article ID 6423974, 15 pages, 2018.

[34] A. Ali, F. Rabiei, and K. Shah, "On Ulam's type stability for a class of impulsive fractional differential equations with nonlinear integral boundary conditions," The Journal of Nonlinear Sciences and Applications, vol. 10, no. 9, pp. 4760-4775, 2017.

[35] M. Benchohra and S. Bouriah, "Existence and stability results for nonlinear boundary value problem for implicit differential equations of fractional order," Moroccan Journal of Pure and Applied Analysis, vol. 1, no. 1, pp. 22-37, 2015.

[36] H. Khan, C. Tunc, W. Chen, and A. Khan, "Existence theorems and hyers-ulam stability for a class of hybrid fractional differential equations with p-laplacian operator," Journal of Applied Analysis and Computation, vol. 8, no. 4, pp. 12111226, 2018.

[37] J. Wang, M. Fečkan, and Y. Tian, "Stability analysis for a general class of non-instantaneous impulsive differential equations"' Mediterranean Journal of Mathematics, vol. 14, no. 2, p. 46, 2017.

[38] J. Wang, K. Shah, and A. Ali, "Existence and hyers-ulam stability of fractional nonlinear impulsive switched coupled evolution equations," Mathematical Methods in the Applied Sciences, vol. 41, no. 6, pp. 2392-2402, 2018.
[39] H. Khan, Y. Li, W. Chen, D. Baleanu, and A. Khan, "Existence theorems and hyers-ulam stability for a coupled system of fractional differential equations with p-laplacian operator," Boundary Value Problems, vol. 2017, no. 1, pp. 1-16, 2017.

[40] K. Deimling, Nonlinear Functional Analysis, Springer, New York, NY, USA, 1985.

[41] M. U. Rehman and R. A. Khan, "Existence and uniqueness of solutions for multi-point boundary value problems for fractional differential equations"' Applied Mathematics Letter, vol. 23, no. 9, p. 239, 2010. 